\title{
Analytical method for the evaluation of field modulation inside the rf-shielded chamber with a time-dependent dipole magnetic field
}

\author{
Yoshihiro Shobuda \\ Japan Atomic Energy Agency, JAEA, 2-4 Shirakata Shirane, Tokaimura, Nakagun, Ibaraki 319-1195, Japan \\ Yoshiro Irie and Susumu Igarashi \\ High Energy Accelerator Research Organization, KEK, 1-1 Oho, Tsukuba, Ibaraki 305-0801, Japan
}

(Received 25 March 2008; published 12 March 2009)

A ceramic chamber with $\mathrm{Cu}$ stripes is usually used as the vacuum chamber in a rapid cycling synchrotron. The $\mathrm{Cu}$ stripes terminate at either end as capacitors, and provide the low impedance for the circulating beam, and the high impedance for the induced current with the frequency components of the external time-dependent magnetic field (for example, injection bump magnets, bending magnets, etc.). It is important to be able to precisely estimate the field modulations inside the chamber when the field is excited, because any such field modulations can cause the beam characteristics to deteriorate. In this paper a theoretical approach to evaluate the field modulations in a quick and precise manner is developed.

DOI: 10.1103/PhysRevSTAB.12.032401

PACS numbers: 03.50.De, 41.85.Ar, 29.27.-a

\section{INTRODUCTION}

Reducing the amount of total magnetic field errors in an accelerator has recently become of importance, because they can result in difficulty in injecting linac beams into the ring and also cause intolerably large closed orbit distortion (COD). The intensity of the proton beam in the accelerator increases [such as that used at the Japan Proton Accelerator Research Complex (J-PARC), the Spallation Neutron Source (SNS), and ISIS [1-3]], thus making it essential to avoid any beam loss caused by magnetic field errors. The linac beam is usually injected into the ring, while the bump (dipole) magnets are excited during the injection period [4-6]. The bump orbit, which is created by the injection bump magnets, has flattop time in preparation for the injection beam. When the uniformity of the flattop field "inside the chamber" is intolerably broken, the beam may be lost during the injection period.

In the rapid cycling synchrotron (RCS), a ceramic chamber covered in $\mathrm{Cu}$ stripes equipped with capacitors is used as the vacuum chamber [7-10]. The capacitance is determined by the low impedance for the circulating beam and the high impedance for the induced current with the frequency components of the time-dependent magnetic field $[7,8]$. The $\mathrm{Cu}$ stripes are necessary in preventing the electromagnetic field from radiating to the external world, when the beam passes through the chamber (and hence is sometimes referred to as an rf-shielded chamber) [9]. If there were no $\mathrm{Cu}$ stripes, the radiation could cause noise in the monitors, which are right besides the chamber, or it could be a big source of impedance to the beam [9-11].

It is evident that the magnetic field inside the ceramic chamber is not the same as without it, when the timedependent magnetic field is excited outside the chamber, because the rf-shielded $\mathrm{Cu}$ stripes interact with the magnetic flux. It is very important to determine whether the field error or field modulation due to this effect is significant, as the possibility exists that any field modulations could cause the characteristics of the beam to deteriorate.

While a standard approach to such a typical accelerator design problem is numerical, theoretical ways of estimating the field modulation inside the chamber would be very useful, and hence in this paper we develop a theory to use when a dipole magnetic field is excited outside the chamber. The theory can also be used to describe the effect of the ferromagnetic yoke surrounding it. In Sec. II, a general theory is derived by considering the interaction between the rf-shielded chamber and the magnetic field. The induced current on the $\mathrm{Cu}$ stripes is classified into two types by source: one being the current in the case where the magnetic flux traverses a $\mathrm{Cu}$ stripe itself, while the other is where the magnetic flux traverses the area enclosed by the pairs of the $\mathrm{Cu}$ stripes and the flanges on both sides of the chamber. In Sec. II, the field modulation deriving from these two types of current are considered. In Sec. III, the developed theory is applied in some simple cases (the bump magnet during the injection period and the bending magnet in the RCS) and explicitly calculate the modulation of the magnetic field inside the chamber. Section IV summarizes this paper.

\section{THEORETICAL WAY OF EVALUATING THE FIELD MODULATION INSIDE THE CHAMBER}

The ceramic chambers in an RCS are usually covered in $\mathrm{Cu}$ stripes equipped with capacitors, as given in Fig. 1. The interaction between the rf-shielded chamber and the dipole magnetic field, which is excited outside of it, was consid- 


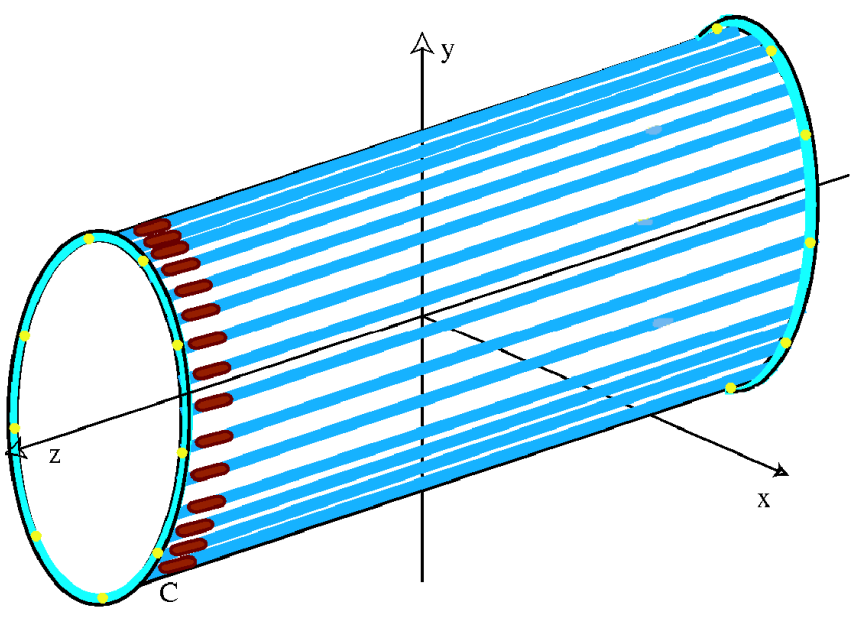

FIG. 1. (Color) The ceramic chamber covered in $\mathrm{Cu}$ stripes equipped with capacitors. There are flanges on both sides of the chamber to fix it along the ring. The brown objects denote capacitors, which are associated on $\mathrm{Cu}$ stripes to realize the high impedance for the induced current with the frequency components of the external time-dependent magnetic field and the low impedance for the circulating beam. This kind of chamber is usually used in a rapid cycling synchrotron (RCS).

ered. Two types of induced current flow in the $\mathrm{Cu}$ stripes: one is the current that flows inside the $\mathrm{Cu}$ stripe, while the other flows between them. The first is caused by the magnetic flux that traverses the $\mathrm{Cu}$ stripe itself, while the other by the flux that traverses the area encircled by the pairs of $\mathrm{Cu}$ stripes and the flanges on both sides of the chamber. In Sec. II, these two types of field modulations are respectively considered. The former is studied in Sec. II A and the latter in Sec. II B.

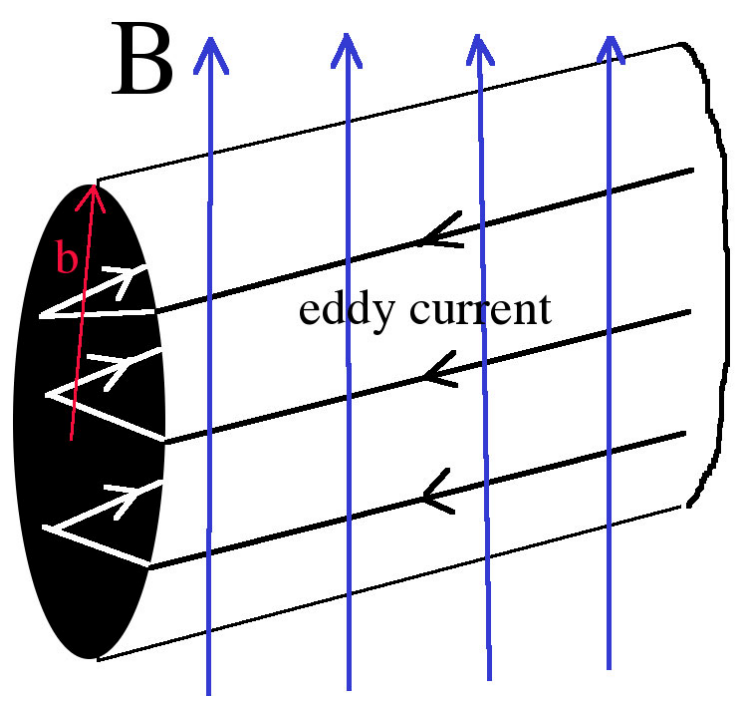

FIG. 2. (Color) The schematic picture of the eddy current, which flows in one of $\mathrm{Cu}$ stripes. For simplicity, it is assumed that the $\mathrm{Cu}$ stripe is cylindrical with its radius $b$. The external magnetic field $B$ traverses the stripe vertically. Current lines couple to each other by the mutual inductance.

\section{A. Effect of the eddy currents caused by the magnetic flux traversing $\mathrm{Cu}$ stripes themselves}

When the magnetic field traverses one of the $\mathrm{Cu}$ stripes, an eddy current flow is generated (refer to Fig. 2), causing a field modulation inside the chamber. In this subsection, this phenomenon will be investigated.

The radius of the cylindrical rf-shielded chamber is given by $a$. For the sake of simplicity, the effects of the ceramics and both edges of this chamber are neglected (this simplification was applied in order to include the after effect of the yoke surrounding the chamber in our theory). Each of the stripes is cylindrical with radius $b$, as depicted in Figs. 2 and 3. (The shape of the $\mathrm{Cu}$ stripes being rectangular will be briefly discussed at the end of this subsection.) As given in Fig. 3, the chamber is surrounded by a ferromagnetic yoke and a primary magnetic field is excited outside of it using current conductors. For example, the ceramic chamber during the injection period with the $3 \mathrm{GeV}$ RCS of J-PARC is just inside a window-frame-type magnet rather than a $C$-type or $H$-type magnet [12]. The assumption was made that $\mathrm{Cu}$ stripes were symmetric to the $x=0$ plane. Each of the stripes was defined

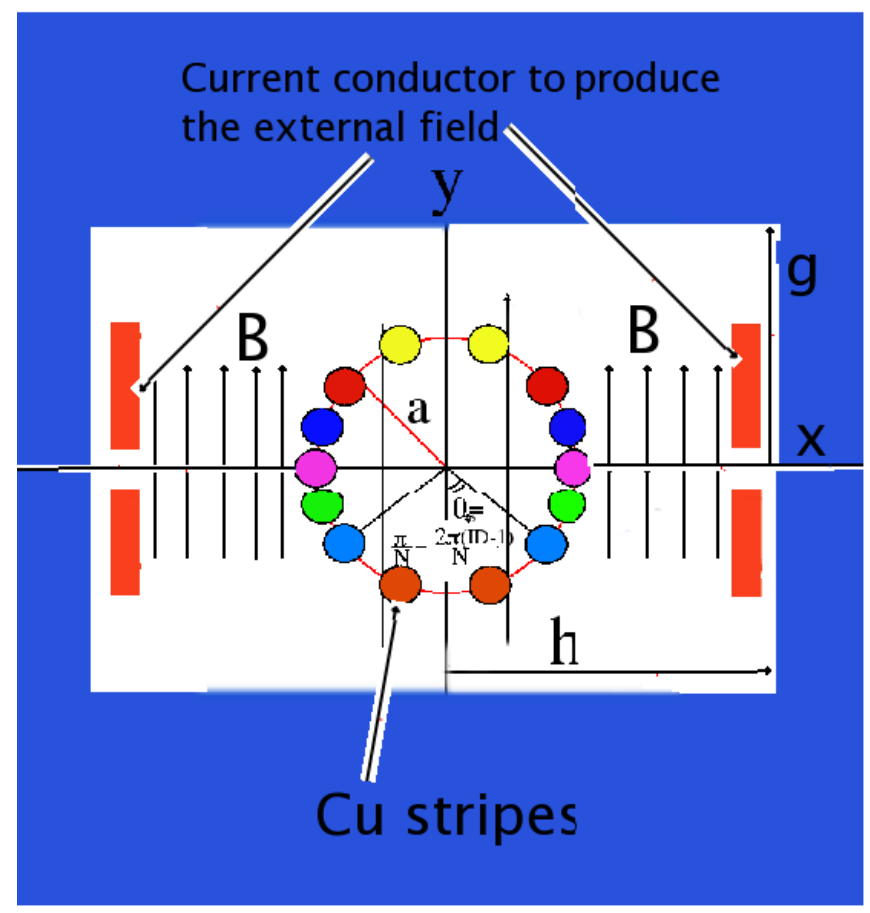

FIG. 3. (Color) The schematic picture of the rf-shielded chamber, which is surrounded by the ferromagnetic yoke that is denoted by the blue object. The current conductors excite the external magnetic field $B(t)$. Cu stripes are symmetric to the $x=$ 0 plane. Each of the stripes is defined by $\left( \pm a \sin \left(\frac{\pi}{N}+\right.\right.$ $\left.\left.\frac{2 \pi(\mathrm{ID}-1)}{N}\right),-a \cos \left(\frac{\pi}{N}+\frac{2 \pi(\mathrm{ID}-1)}{N}\right), z\right)$, with ID used as the index to specify the IDth stripe that runs from 1 to $N / 2, N$ is the total number of stripes, $a$ is the radius of chamber, $2 g$ is the gap height of yoke, and $2 h$ is the gap width of yoke. The direction of the external magnetic field is vertical $y$. 
by $\left( \pm a \sin \theta_{\mathrm{ID}},-a \cos \theta_{\mathrm{ID}}, z\right)$, where $\theta_{\mathrm{ID}}=\frac{\pi}{N}+\frac{2 \pi(\mathrm{ID}-1)}{N}$, with ID used as the index, which runs from 1 to $N / 2$, to specify the IDth stripe and $N$ the total number of $\mathrm{Cu}$ stripes.

Since all stripes are cylindrical, the position of the eddy current was specified using both a local coordinate $(r, \varphi, z)$ and the global coordinate $(x, y, z)$ used to identify the $\mathrm{Cu}$ stripe (refer to Fig. 4). The angle $\varphi$ moves from $-\pi$ to $\pi$. The symmetry of this system reveals that the direction of the eddy current for $0<\varphi<\pi$ and that for $-\pi<\varphi<0$ are opposites. Using this property, we imagined a small coil inside the stripe that is composed of the lines at $(r \sin \varphi, r \cos \varphi, z)$ and $(-r \sin \varphi, r \cos \varphi, z)$.

The inductance between the coils specified with the coordinates of $(r, \varphi, z)$ and $\left(r^{\prime}, \varphi^{\prime}, z\right)$ can now be calculated. Ampere's law gives us the magnetic field on the coil composed of the lines $\left(r^{\prime} \sin \varphi^{\prime}, r^{\prime} \cos \varphi^{\prime}, z\right)$ and $\left(-r^{\prime} \sin \varphi^{\prime}, r^{\prime} \cos \varphi^{\prime}, z\right)$ when caused by eddy currents at $(r \sin \varphi, r \cos \varphi, z)$ and $(-r \sin \varphi, r \cos \varphi, z)$. The mutual inductance $m_{12}$ between the coils is then given by

$$
m_{12}=\frac{Z_{0}}{2 \pi c} \log \frac{r^{2}+r^{\prime 2}-2 r r^{\prime} \cos \left(\varphi^{\prime}+\varphi\right)}{r^{2}+r^{\prime 2}-2 r r^{\prime} \cos \left(\varphi^{\prime}-\varphi\right)},
$$

where $Z_{0}(=120 \pi)$ is the impedance of the free space and $c$ is the velocity of light.

In order to obtain the current density $i(r, \varphi)$ of the coil, which is composed of the lines $(r \sin \varphi, r \cos \varphi, z)$ and $(-r \sin \varphi, r \cos \varphi, z)$, a circuit equation needs to be created. Not only the effect of the resistance of this coil, but also the effect of another coil specified by $\left(r^{\prime}, \varphi^{\prime}\right)$ needs to be

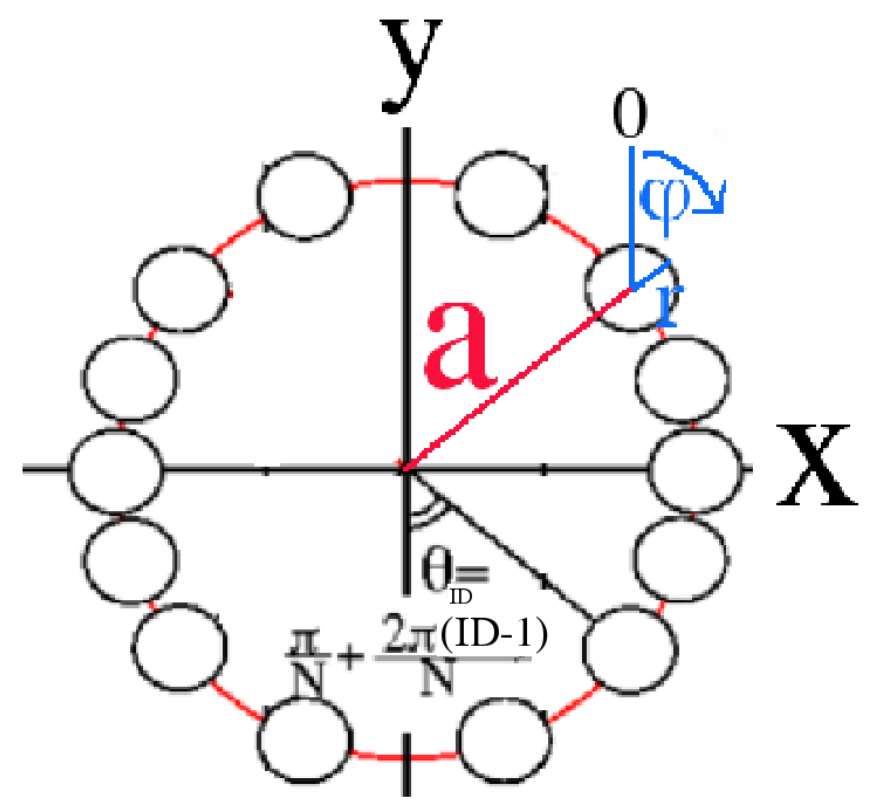

FIG. 4. (Color) The global coordinate and the local coordinate. The global coordinate $(x, y, z)$ specifies the $\mathrm{Cu}$ stripe and the local coordinate $(r, \varphi, z)$ specifies the position of the eddy current in one of $\mathrm{Cu}$ stripes. included in this equation. Since the formula of the inductances $m_{12}$ is given by Eq. (1), the circuit equation can be described as follows:

$$
\begin{aligned}
\frac{d B}{d t} 2 r \sin \varphi= & \int_{0}^{b} d r^{\prime} \int_{0}^{\pi} d \varphi^{\prime} r^{\prime} \frac{d i\left(r^{\prime}, \varphi^{\prime}\right)}{d t} \frac{Z_{0}}{2 \pi c} \\
& \times \log \frac{r^{2}+r^{\prime 2}-2 r r^{\prime} \cos \left(\varphi^{\prime}+\varphi\right)}{r^{2}+r^{\prime 2}-2 r r^{\prime} \cos \left(\varphi^{\prime}-\varphi\right)} \\
& +\frac{2 i(r, \varphi)}{\sigma},
\end{aligned}
$$

where $\sigma$ is the conductivity of copper and $B$ the original magnetic flux density, which was assumed to be uniform spatially for the sake of simplicity. In the above description, the assumption was also made that the field modulation to be derived can be dealt in a perturbative manner, when compared to the original magnetic flux. It will be shown that this assumption is plausible in Sec. III when the field modulation is actually calculated.

In order to solve Eq. (2), the assumption was made that current density $i(r, \varphi)$ can be expanded as

$$
i(r, \varphi)=\sum_{k=0}^{\infty} \lambda_{k} r^{2 k+1} \sin \varphi,
$$

considering the symmetry of this system. Substituting Eq. (3) into Eq. (2) and integrating it for $r^{\prime}$ and $\varphi^{\prime}$ results in

$$
\begin{gathered}
\frac{d B}{d t}=\frac{Z_{0}}{4 c} \sum_{k=0}^{\infty} \frac{d \lambda_{k}}{d t} \frac{b^{2 k+2}}{k+1}+\frac{\lambda_{0}}{\sigma}, \\
\lambda_{k+1}=\frac{Z_{0} \sigma}{4 c} \frac{d \lambda_{k}}{d t}\left(\frac{1}{k+1}-\frac{1}{k+2}\right) \\
=\prod_{i=0}^{k}\left(\frac{1}{i+1}-\frac{1}{i+2}\right)\left(\frac{Z_{0} \sigma}{4 c}\right)^{k+1} \frac{d^{k+1} \lambda_{0}}{d t^{k+1}} .
\end{gathered}
$$

Here the fact that Eq. (2) should be satisfied for arbitrary $r$ and $\varphi$ was used, because variable $r$ moves in $0<r<b$ while $\varphi$ does in $-\pi<\varphi<\pi$. Substituting Eq. (5) into Eq. (4) results in an infinite order differential equation for $\lambda_{0}$. While this is rather difficult to solve, for the special case satisfying the following condition,

$$
\frac{Z_{0} \sigma b^{2}}{4 c T_{0}}<1
$$

(time $T_{0}$ is the typical time scale for the change in magnetic flux $B$ ), this equation can be truncated by the first order differential of $\lambda_{0}$. Since this condition can be satisfied for almost every case, the infinite order differential equation turns out to be the first order differential equation:

$$
\frac{d B(t)}{d t}=\frac{\lambda_{0}}{\sigma}+\frac{Z_{0} b^{2}}{4 c} \frac{d \lambda_{0}}{d t} .
$$

By solving Eq. (7), the formula for the current density can be obtained as 


$$
i(r, \varphi)=\frac{4 c}{Z_{0} b^{2}} \int_{-\infty}^{t} d t^{\prime} \frac{d B\left(t^{\prime}\right)}{d t^{\prime}} e^{\left[(4 c) /\left(Z_{0} b^{2} \sigma\right)\right]\left(t^{\prime}-t\right)} r \sin \varphi .
$$

Since we know the current density of a stripe, the field on the axis of the chamber can be calculated using Ampere's law. At first, the effect of one $\mathrm{Cu}$ stripe specified by $\left(a \sin \theta_{i},-a \cos \theta_{i}, z\right)$ is calculated, and then, the effect of all stripes is superposed. For this purpose, it is convenient to use both local and global coordinates. Since the magnetic field at the center $(x=0, y=0, z)$ of the chamber, which comes from $i$ th stripe, can be described as

$$
\begin{aligned}
H_{i} & =\frac{2 c}{\pi b^{2} Z_{0}} \int_{-\infty}^{t} d t^{\prime} \frac{d B\left(t^{\prime}\right)}{d t^{\prime}} e^{\left[(4 c) /\left(Z_{0} b^{2} \sigma\right)\right]\left(t^{\prime}-t\right)} \int_{0}^{b} d r \oint d \varphi \frac{r^{2} \sin \varphi\left(-a \cos \theta_{i}+r \cos \varphi,-a \sin \theta_{i}-r \sin \varphi, 0\right)}{a^{2}+r^{2}-2 a r \cos \left(\theta_{i}+\varphi\right)} \\
& =\frac{c b^{2}}{2 a^{2} Z_{0}} \int_{-\infty}^{t} d t^{\prime} \frac{d B\left(t^{\prime}\right)}{d t^{\prime}} e^{\left[(4 c) /\left(Z_{0} b^{2} \sigma\right)\right]\left(t^{\prime}-t\right)}\left(\sin 2 \theta_{i},-\cos 2 \theta_{i}, 0\right),
\end{aligned}
$$

using the formulas

$$
\begin{gathered}
\oint d \varphi \frac{\sin \varphi(-a \cos \theta+r \cos \varphi)}{a^{2}+r^{2}-2 a r \cos (\theta+\varphi)}=\frac{\pi r}{a^{2}} \sin 2 \theta, \\
\oint d \varphi \frac{\sin \varphi(-a \sin \theta-r \sin \varphi)}{a^{2}+r^{2}-2 a r \cos (\theta+\varphi)}=-\frac{\pi r}{a^{2}} \cos 2 \theta,
\end{gathered}
$$

the total effect of all the stripes can be summarized as

$$
\begin{aligned}
\Delta B_{y}= & -\frac{b^{2}}{a^{2}} \int_{-\infty}^{t} d t^{\prime} \frac{d B\left(t^{\prime}\right)}{d t^{\prime}} e^{\left[(4 c) /\left(Z_{0} b^{2} \sigma\right)\right]\left(t^{\prime}-t\right)} \sum_{i=1}^{N / 2} \cos 2 \theta_{i} \\
= & -\frac{b^{2}}{2} \int_{-\infty}^{t} d t^{\prime} \frac{d B\left(t^{\prime}\right)}{d t^{\prime}} e^{\left[(4 c) /\left(Z_{0} b^{2} \sigma\right)\right]\left(t^{\prime}-t\right)} \\
& \times \sum_{i=1}^{N} \frac{y_{i}^{2}-x_{i}^{2}}{\left(y_{i}^{2}+x_{i}^{2}\right)^{2}}
\end{aligned}
$$

by applying the law of superposition in Eq. (9).

Now, the case where the ferromagnetic yoke surrounds the chamber is considered. When the relative permeability of magnetic yoke $\mu^{\prime}$ is infinite, the magnetic field lines must be perpendicular to the face of the magnetic pole. This situation can be realized by using image currents of $\left[2 m h+(-1)^{m} a \sin \theta_{i}, 2 n g-(-1)^{n} a \cos \theta_{i}, z\right]$ instead of the ferromagnetic yoke, where $2 g$ is the gap height of the magnet yoke, $2 h$ the gap width of the yoke, and $m$ and $n$ are arbitrary integers (refer to Appendix A and Fig. 5). Since the current density $i(r, \varphi)$ is antisymmetric in the transformation of $\varphi \rightarrow-\varphi$, the direction of the current should be opposite each time integer $m$, which specifies the horizontal position of the image current, differs [i.e. the direction of the current at $\left[2 m h+(-1)^{m} a \sin \theta_{i}\right.$, $\left.2 n g-(-1)^{n} a \cos \theta_{i}, z\right] \quad$ and at $\quad[2(m+1) h+$ $\left.(-1)^{m+1} a \sin \theta_{i}, 2 n^{\prime} g-(-1)^{n^{\prime}} a \cos \theta_{i}, z\right]$ are opposite, while integers $n$ and $n^{\prime}$ are not necessarily the same] but, the direction of the current is the same vertically for fixed integer $m$. We finally obtain the expression for the field modulation on the axis of the rf-shielded chamber inside the ferromagnetic yoke as

$$
\begin{aligned}
\Delta B_{y, \text { ferr }} & =\int_{-\infty}^{t} d t^{\prime} \frac{d B\left(t^{\prime}\right)}{d t^{\prime}} e^{\left[(4 c) /\left(Z_{0} b^{2} \sigma\right)\right]\left(t^{\prime}-t\right)} b^{2} \sum_{i=1}^{N / 2} \sum_{m, n=-\infty}^{\infty}(-1)^{m} \frac{\left[2 m h+(-1)^{m} a \sin \theta_{i}\right]^{2}-\left[2 n g-(-1)^{n} a \cos \theta_{i}\right]^{2}}{\left\{\left[2 m h+(-1)^{m} a \sin \theta_{i}\right]^{2}+\left[2 n g-(-1)^{n} a \cos \theta_{i}\right]^{2}\right\}^{2}} \\
& =\int_{-\infty}^{t} d t^{\prime} \frac{d B\left(t^{\prime}\right)}{d t^{\prime}} e^{\left[(4 c) /\left(Z_{0} b^{2} \sigma\right)\right]\left(t^{\prime}-t\right)} \cdot \frac{b^{2}}{2} \sum_{i=1}^{N} \sum_{m, n=-\infty}^{\infty}(-1)^{m} \frac{\left[2 m h+(-1)^{m} x_{i}\right]^{2}-\left[2 n g+(-1)^{n} y_{i}\right]^{2}}{\left\{\left[2 m h+(-1)^{m} x_{i}\right]^{2}+\left[2 n g+(-1)^{n} y_{i}\right]^{2}\right\}^{2}}
\end{aligned}
$$

Equation (13) shows that the time dependence and the geometrical dependence in field modulation $\Delta B_{y, \text { ferr }}$ is totally divided, or describable as the product of the two factors. It was also discovered that the relaxation time $\tau$ for the damping of this field modulation can be characterized using

$$
\tau=\frac{Z_{0} b^{2} \sigma}{4 c} .
$$

This reflects the fact that the $\mathrm{Cu}$ stripe itself has its own inductance.
When the shape of the stripe is rectangular (which is specified using $2 b_{x} \times 2 b_{y}$ ), it is much more difficult to obtain a formula for current density $i(\xi$, $\eta)$, where $(\xi, \eta)$ is the local Cartesian coordinate of a stripe. Considering the property that the current density should be antisymmetric with respect to the $\xi=0$ plane, the simple assumption is made that $i(\xi, \eta)$ can be approximated using

$$
i(\xi, \eta)=\frac{4 c}{Z_{0} b_{x}^{2}} \int_{-\infty}^{t} d t^{\prime} \frac{d B\left(t^{\prime}\right)}{d t^{\prime}} e^{\left[(4 c) /\left(Z_{0} b_{x}^{2} \sigma\right)\right]\left(t^{\prime}-t\right)} \xi,
$$

by referring to Eq. (8) where the shape of the stripe was 
cylindrical. In the above expression the radius of stripe $b$ is replaced by the horizontal half length of rectangular stripe $b_{x}$, because magnetic flux $B$ traverses the stripe vertically.
Similar to the cylindrical case, the field modulation at the center of the chamber due to the $i$ th stripe that exists at $\left(X_{i}, Y_{i}\right)$ can be described using

$$
\begin{gathered}
\Delta B_{i, y}=-\frac{2}{\pi b_{x}^{2}} \int_{-\infty}^{t} d t^{\prime} \frac{d B\left(t^{\prime}\right)}{d t^{\prime}} e^{\left[(4 c) /\left(Z_{0} b_{x}^{2} \sigma\right)\right]\left(t^{\prime}-t\right)} \int_{-b_{x}}^{b_{x}} d \xi \int_{-b_{y}}^{b_{y}} d \eta \frac{\left(X_{i}+\xi\right) \xi}{\left(X_{i}+\xi\right)^{2}+\left(Y_{i}+\eta\right)^{2}} \\
=\frac{2}{\pi b_{x}^{2}} \int_{-\infty}^{t} d t^{\prime} \frac{d B\left(t^{\prime}\right)}{d t^{\prime}} e^{\left[(4 c) /\left(Z_{0} b_{x}^{2} \sigma\right)\right]\left(t^{\prime}-t\right)} Q\left(X_{i}, Y_{i}\right), \\
Q\left(X_{i}, Y_{i}\right)=-\frac{b_{x}^{2}-X_{i}^{2}}{2} \tan ^{-1} \frac{Y+b_{y}}{X+b_{x}}+\frac{\left(Y+b_{y}\right)^{2}}{2} \tan ^{-1} \frac{X+b_{x}}{Y+b_{y}}+\frac{b_{x}^{2}-X_{i}^{2}}{2} \tan ^{-1} \frac{Y+b_{y}}{X-b_{x}}-\frac{\left(Y+b_{y}\right)^{2}}{2} \tan ^{-1} \frac{X-b_{x}}{Y+b_{y}} \\
+\frac{b_{x}^{2}-X_{i}^{2}}{2} \tan ^{-1} \frac{Y-b_{y}}{X+b_{x}}-\frac{\left(Y-b_{y}\right)^{2}}{2} \tan ^{-1} \frac{X+b_{x}}{Y-b_{y}}-\frac{b_{x}^{2}-X_{i}^{2}}{2} \tan ^{-1} \frac{Y-b_{y}}{X-b_{x}}+\frac{\left(Y-b_{y}\right)^{2}}{2} \tan ^{-1} \frac{X-b_{x}}{Y-b_{y}} \\
-2 b_{x} b_{y}+\frac{X_{i}\left(Y_{i}+b_{y}\right)}{2} \log \frac{\left(X+b_{x}\right)^{2}+\left(Y+b_{y}\right)^{2}}{\left(X-b_{x}\right)^{2}+\left(Y+b_{y}\right)^{2}}-\frac{X_{i}\left(Y_{i}-b_{y}\right)}{2} \log \frac{\left(X+b_{x}\right)^{2}+\left(Y-b_{y}\right)^{2}}{\left(X-b_{x}\right)^{2}+\left(Y-b_{y}\right)^{2}} .
\end{gathered}
$$

When the ferromagnetic yoke surrounds the rf-shielded chamber, the above formula can be generalized as

$$
\Delta B_{y, \text { ferr }}=\frac{2}{\pi b_{x}^{2}} \int_{-\infty}^{t} d t^{\prime} \frac{d B\left(t^{\prime}\right)}{d t^{\prime}} e^{\left[(4 c) /\left(Z_{0} b_{x}^{2} \sigma\right)\right]\left(t^{\prime}-t\right)} \sum_{i=1}^{N} \sum_{m, n=-\infty}^{\infty}(-1)^{m} Q\left[2 m h+(-1)^{m} X_{i}, 2 n g+(-1)^{n} Y_{i}\right],
$$

by summing the effects of the image currents, instead of the ferromagnetic yoke.

\section{B. Effects of induced currents caused by the magnetic flux traversing the area enclosed by the pair of $\mathrm{Cu}$ stripes and flanges on both sides of the chamber}

When the magnetic field traverses the area enclosed by the pair of $\mathrm{Cu}$ stripes and flanges on both sides of the

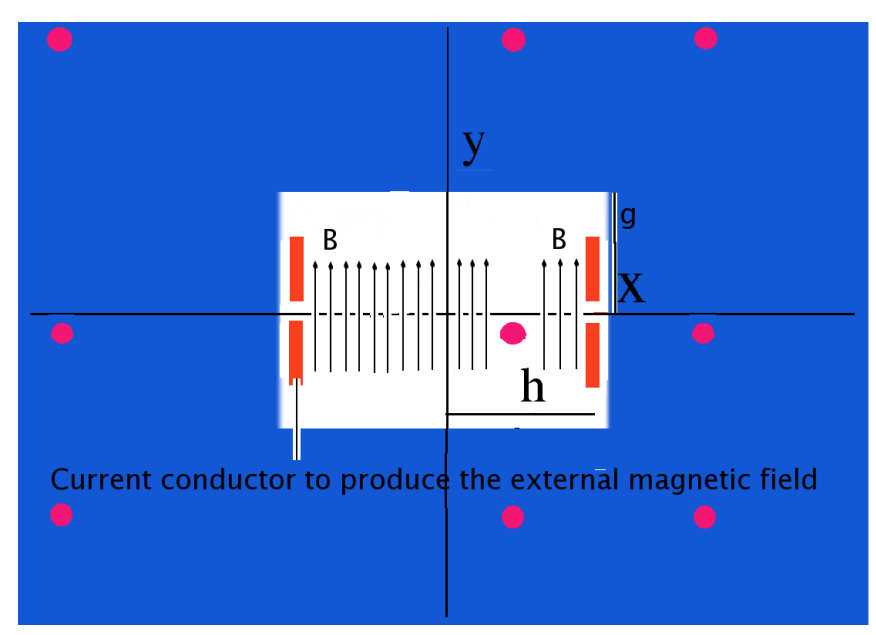

FIG. 5. (Color) The effect of the ferromagnetic yoke, which is denoted by the blue object, is replaced by that of the image currents that are represented by pink objects. The image currents are located at $\left[2 m h+(-1)^{m} a \sin \theta_{i}, 2 n g-(-1)^{n} a \cos \theta_{i}, z\right]$, where $m$ and $n$ are arbitrary integers. The orange objects denote the current conductors to produce the external magnetic field $B(t)$. chamber, the induced current flows over all the stripes. It is essential to take this effect into account to evaluate the field modulation correctly, because the flanges on both sides of the chamber connects the $\mathrm{Cu}$ stripes electrically in reality, and the induced currents flow over all the stripes interactively. In this subsection, the field modulation inside the chamber due to this effect will be considered.

As given in Fig. 3, since each stripe is at $\left( \pm a \sin \theta_{i},-a \cos \theta_{i}, z\right)$ the system of $N \mathrm{Cu}$ stripes was simplified into that of $N / 2$ coils by combining the $i$ th and $-i$ th stripes and flanges on both sides of the chamber (refer to the left in Fig. 6). In order to take into account the effect of the yoke in our theory, the assumption was made
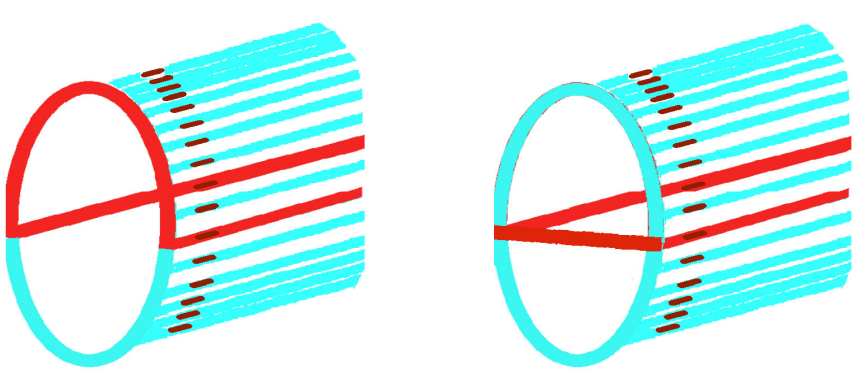

FIG. 6. (Color) The schematic picture of the $i$ th $\mathrm{Cu}$ coil, which is denoted by the red line. The brown objects denote capacitors. The coil is originally defined by combining the $i$ th and $-i$ th $\mathrm{Cu}$ stripes and the flanges on both sides of the chamber as shown in the left figure. As a result of applying Gauss's law in one of Maxwell equations $(\operatorname{div} B=0)$, the form of this coil can be deformed to that in the right figure, because the longitudinal component of magnetic field is assumed to be zero. 
that the longitudinal component of the magnetic fields could be neglected. This simplification enabled changing the form of the coil given on the left in Fig. 6 to that given on the right, as a result of applying Gauss's law in one of Maxwell equations ( $\operatorname{div} B=0)$. From here on in, we simply collectively call the $i$ th coil that which is composed of the $i$ th and $-i$ th stripes. Using this simplification, the system of an rf-shielded chamber in an excited dipole magnetic field is rewritten to that of multicoils. The appropriateness of this simplification is discussed by comparing our results with simulated results using OPERA-3D ELEKTRA in the next section [13].

The magnetic field at $(x, y, z)$ needs to be known when current $I$ flows on the $i$ th coil. When intrinsic frequency $f$ of the current in this coil is sufficiently lower than that of factor $\sigma c Z_{0} / 2 \pi$, the displacement current can be ignored in the Maxwell equation, and can then be evaluated using Ampere's law. Since copper is used in the stripe that shields the electromagnetic field, it can be evaluated as $\sigma c Z_{0} / 2 \pi=1.0 \times 10^{18} \mathrm{~Hz}$. Since this value is usually sufficiently higher than the normal mode frequencies of coils (for example, refer to Fig. 32 in the next section), this simplification was applied. As the assumption was made that the longitudinal component of magnetic fields could be ignored, only the situation was considered where current $I$ flows at $\left(a \sin \theta_{i},-a \cos \theta_{i}, z\right)$ and $-I$ at $\left(-a \sin \theta_{i},-a \cos \theta_{i}, z\right)$ inside the ferromagnetic yoke, and hence the induced magnetic field $H$ at $(x, y, z)$ can be written:

$$
\begin{aligned}
\mathbf{H}= & \sum_{m, n=-\infty}^{\infty} \frac{I\left\{-\left[y-2 n g+(-1)^{n} a \cos \theta_{i}\right], x-2 m h-(-1)^{m} a \sin \theta_{i}, 0\right\}}{2 \pi\left\{\left[x-2 m h-(-1)^{m} a \sin \theta_{i}\right]^{2}+\left[y-2 n g+(-1)^{n} a \cos \theta_{i}\right]^{2}\right\}} \\
& -\sum_{m, n=-\infty}^{\infty} \frac{I\left\{-\left[y-2 n g+(-1)^{n} a \cos \theta_{i}\right], x-2 m h+(-1)^{m} a \sin \theta_{i}, 0\right\}}{2 \pi\left\{\left[x-2 m h+(-1)^{m} a \sin \theta_{i}\right]^{2}+\left[y-2 n g+(-1)^{n} a \cos \theta_{i}\right]^{2}\right\}},
\end{aligned}
$$

where the effect of the ferromagnetic yoke is replaced by the image currents at $\left[2 m h+(-1)^{m} a \sin \theta_{i}, 2 n g-\right.$ $\left.(-1)^{n} a \cos \theta_{i}, z\right] \quad$ and $\quad\left[2 m h-(-1)^{m} a \sin \theta_{i}, 2 n g-\right.$ $\left.(-1)^{n} a \cos \theta_{i}, z\right]$ (refer to Fig. 5). Differing from the case in the previous subsection, the direction of the current, specified by integers $m$ and $n$, is the same for a different $m$ and $n$ when deriving Eq. (19).

Since each coil interacts through mutual inductances, it is necessary to calculate, in advance, the self and mutual inductance between the $i$ th and $j$ th coils to obtain the current for each coil. The magnetic flux crossing the $j$ th coil arriving from the current on the $i$ th coil, can be obtained by the integration of $\mu_{0} \times$ Eq. (19) from $-a \sin \theta_{j}$ to $a \sin \theta_{j}$ over $x$ and from $-l / 2$ to $l / 2$ over $z$ on the fixed $y=-a \cos \theta_{j}$ plane, where $l$ is the longitudinal length of the chamber. The mutual inductance between the $i$ th and $j$ th coil is obtained by dividing this magnetic flux by current $-I$. The self and mutual inductance matrix $L_{i j}$ is then given by

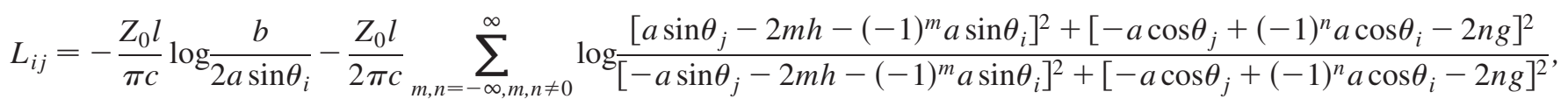

for $i=j$, and

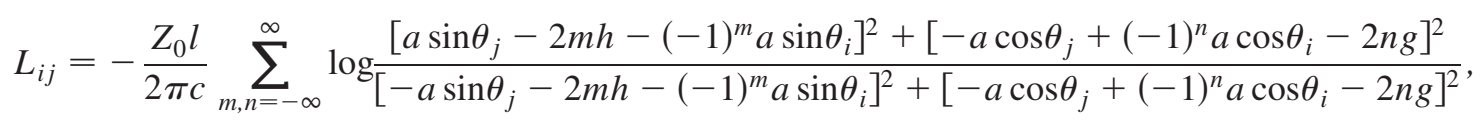

for $i \neq j$, where integers $m$ and $n$ do not become zero simultaneously in the summation of the second term of Eq. (20). When the ferromagnetic yoke does not surround the chamber, the effects of the image currents do not have to be taken into account. Equations (20) and (21) reproduce the well-known formula [14]:

$$
L_{i j}= \begin{cases}-\frac{Z_{0} l}{\pi c} \log _{\frac{1}{2 a \sin \theta_{i}},} & \text { for } i=j, \\ -\frac{Z_{0} l}{2 \pi c} \log \frac{\left(a \sin \theta_{j}-a \sin \theta_{i}\right)^{2}+\left(-a \cos \theta_{j}+a \cos \theta_{i}\right)^{2}}{\left(-a \sin \theta_{j}-a \sin \theta_{i}\right)^{2}+\left(-a \cos \theta_{j}+a \cos \theta_{i}\right)^{2}}, & \text { for } i \neq j .\end{cases}
$$

It should be noticed that inductance matrix $L_{i j}$ given by Eqs. (20)-(22) is symmetric.

The current on each coil will now be calculated using electric circuit theory [15]. When time-dependent dipole magnetic flux density $B(t)$ is excited outside the chamber, current $I_{i}$ on the $i$ th coil (actually consisting of the $i$ th and $-i$ th stripes), is in line with the following equation: 


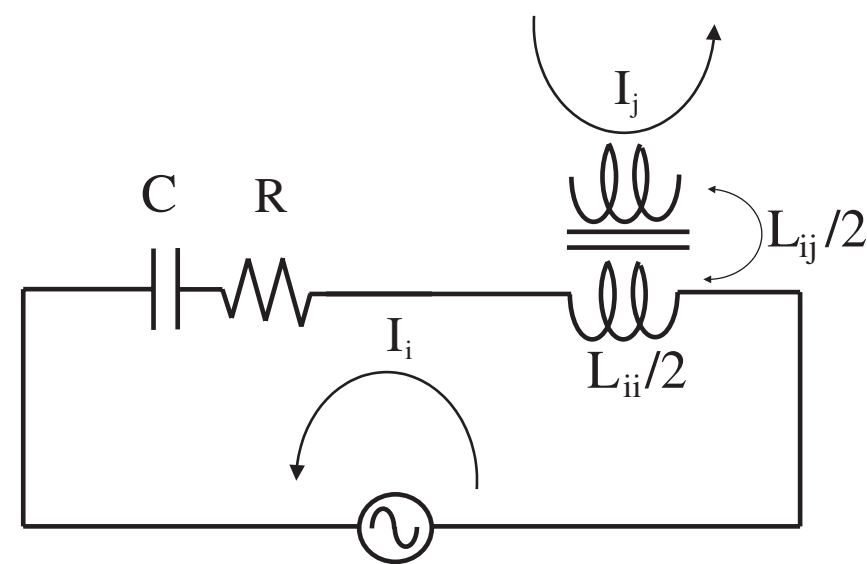

FIG. 7. The schematic picture of the $i$ th $\mathrm{Cu}$ stripe in terms of the electric circuit theory. The character $C$ denotes the capacitor associated on the $\mathrm{Cu}$ stripe, $R$ does the resistance of $\mathrm{Cu}$ stripe, $L_{i j}$ is the mutual inductance between the $i$ th and the $j$ th coil, and $L_{i i}$ is the self-inductance of the $i$ th coil.

$$
B^{\prime \prime}(t) a l \sin \theta_{i}=\sum_{j=1}^{N / 2} \frac{L_{i j}}{2} \frac{d^{2} I_{j}}{d t^{2}}+R \frac{d I_{i}}{d t}+\frac{I_{i}}{C},
$$

where $R$ is the resistance of each $\mathrm{Cu}$ stripe and $C$ the associated capacitance. Here the assumption is made that each stripe can be described using the electric circuit in Fig. 7 and that the influence on the original magnetic flux of the induced current is insignificant. Since the inductance matrix $L_{i j}$ is symmetric, this equation can be diagonalized using the orthogonal matrix $U_{i j}$. When the eigenvalues of the inductance matrix are provided with $\lambda_{i}$, the current on the $i$ th coil can be solved as

$$
\begin{aligned}
I_{i}= & -\sum_{j, k=1}^{N / 2} U_{i j} \int_{-\infty}^{t} d t^{\prime} \frac{i}{\sqrt{\frac{2 \lambda_{j}}{C}-R^{2}}} B^{\prime \prime}\left(t^{\prime}\right) a l U_{j k}^{-1} \\
& \times \sin \theta_{k} e^{\left[\left(R / \lambda_{j}\right)-\left(i / \lambda_{j}\right) \sqrt{\left(2 \lambda_{j} / c\right)-R^{2}}\right]\left(t^{\prime}-t\right)} \\
& +\sum_{j, k=1}^{N / 2} U_{i j} \int_{-\infty}^{t} d t^{\prime} \frac{i}{\sqrt{\frac{2 \lambda_{j}}{C}-R^{2}}} B^{\prime \prime}\left(t^{\prime}\right) a l U_{j k}^{-1} \\
& \times \sin \theta_{k} e^{\left[\left(R / \lambda_{j}\right)+\left(i / \lambda_{j}\right) \sqrt{\left.\left(2 \lambda_{j}\right) / C\right)-R^{2}}\right]\left(t^{\prime}-t\right)} .
\end{aligned}
$$

Once the current of the $i$ th coil is known, the magnetic field on the axis of the chamber can be obtained using the

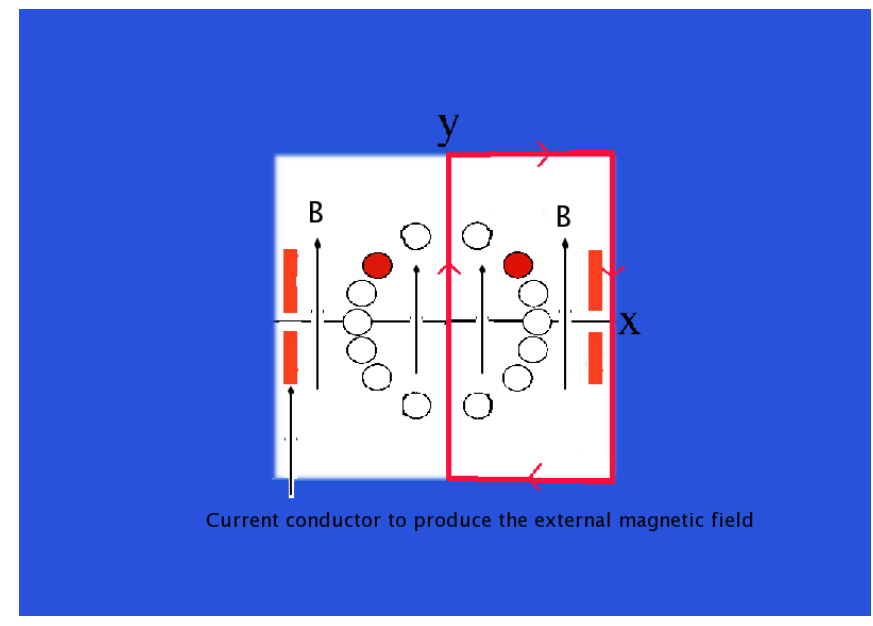

FIG. 8. (Color) The red line denotes the path to which Ampere's law is applied. The approximation is made that the field on the $x=0$ plane is constant in the derivation of Eq. (25). The blue and orange objects represent the ferromagnetic yoke and the current conductors to produce the external magnetic field $B(t)$, respectively.

superposition of the currents of all the stripes. If the assumption is made that the field on the $x=0$ plane is constant, a simple formula for the field modulation $\Delta B_{y}$ can be approximated using

$$
\Delta B_{y}=-\frac{Z_{0}}{2 c g} \sum_{i=1}^{N / 2} I_{i}
$$

as a result of applying Ampere's law to the path depicted in Fig. 8, because the azimuthal component of the magnetic field is zero at the surface of the ferromagnetic yoke with its relative permeability of $\mu^{\prime}=\infty$.

A more rigorous formula can be also obtained by substituting Eq. (24) into Eq. (19) where the effects of the magnet yoke are replaced by those of the image currents. It should be noted here that the contribution of this image current is already included in the inductance matrix formula given using Eqs. (20) and (21). When the magnet yoke surrounds the chamber, the final expression of the modulation of the magnetic flux at the axis of the chamber is

$$
\Delta B_{y}=-\frac{Z_{0}}{\pi c} \sum_{i=1}^{N / 2} \sum_{m, n=-\infty}^{\infty} \frac{(-1)^{m} a I_{i} \sin \theta_{i}\left[-4 m^{2} h^{2}+a^{2}+4 n^{2} g^{2}-4 n(-1)^{n} g a \cos \theta_{i}\right]}{\left[4 m^{2} h^{2}+4 n^{2} g^{2}+a^{2}-4 n g(-1)^{n} a \cos \theta_{i}\right]^{2}-16 m^{2} h^{2} a^{2} \sin ^{2} \theta_{i}} .
$$

By comparing Eq. (25) with Eq. (26), a discussion is made in the next section whether the approximation of the magnetic field being constant on the $x=0$ plane, which was used in deriving Eq. (25), is appropriate or not.

\section{APPLICATION}

In this section, the theory is applied in some simple cases: the bump magnet during the injection period and the bending magnet, in particular for $3 \mathrm{GeV}$ RCS at 


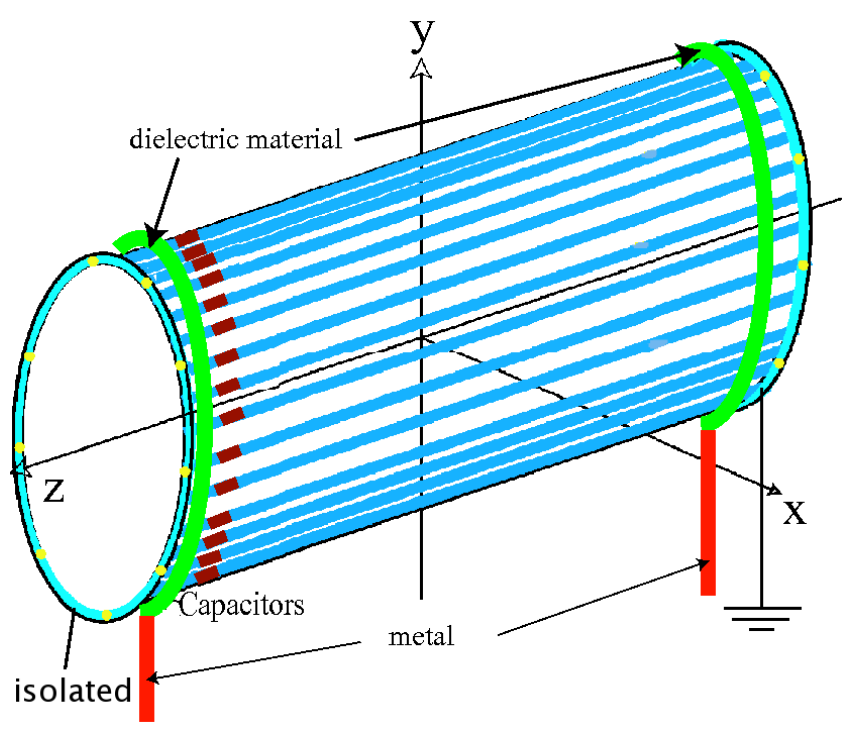

FIG. 9. (Color) The chamber with flanges is fixed by dielectric materials (green objects), which are supported by metal poles (red objects) with the $3 \mathrm{GeV}$ RCS at J-PARC. $\mathrm{Cu}$ stripes terminate at either end as capacitors. One side of the chamber would be approximately isolated, while the other side would act as the Earth

J-PARC. The modulation of the magnetic field inside the chamber is concretely calculated for these cases.

As given in Fig. 9, a chamber with flanges is fixed by dielectric materials, which are supported by metal poles with the $3 \mathrm{GeV}$ RCS at J-PARC. The capacitance made up of the dielectric materials and the $\mathrm{Cu}$ stripes is typically evaluated as being in the tens of $\mathrm{pF}$. Since that value is significantly smaller than the capacitance of the attached capacitors (typically $0.3 \mu \mathrm{F}$ ), the approximation was made that the one side of the chamber would be approximately isolated, while the other side would act as the Earth (because the corresponding flange is grounded).

\section{A. The modulation of the magnetic field inside the chamber when the injection bump magnet is excited}

A linac beam is usually injected into the ring through creating a bump orbit during the injection period. With a high intensity proton machine like that used at J-PARC, it is important to evaluate the influence of the field modulation on the beam due to the interaction between the rfshielded chamber and the original magnetic field. Furthermore, a significant decision must be made with the field pattern of the bump magnet in order that the field modulation does not cause any deterioration in the characteristics of the beam.

\section{The field modulation due to the magnetic flux traversing the $\mathrm{Cu}$ stripe}

First, the field modulation due to the magnetic flux traversing the $\mathrm{Cu}$ stripe is considered. The formulas given in Eqs. (13) and (18) describe this effect. Before exploring the above case where the bump magnet excites the magnet flux, the accuracy of the aforementioned formulas is verified by comparing the theoretical results with the simulated results using OPERA-3D ELEKTRA with a much simpler field pattern, which is given in Fig. 10. The formula given in Eq. (13), which is applicable in the case where the stripes are cylindrical, is more rigorous than that given in Eq. (18), which applies to the rectangular stripe. However, it is rather difficult to deal with a cylindrical stripe using OPERA-3D ELEKTRA. Hence, only a rectangular stripe is considered and the results are compared with those calculated with Eq. (18).

A chamber with radius $a=15.25 \mathrm{~cm}$ was surrounded by a magnet yoke of longitudinal length $80.0 \mathrm{~cm}$, which was the longitudinal length of the chamber, too. The gap height $2 g$ was $32.0 \mathrm{~cm}$ while the gap width $2 h$ is $64.0 \mathrm{~cm}$. In this case the magnetic field was assumed to be excited during $0.5 \mathrm{~ms}$ and its flux density at $x=y=0$ to have become $1664 \mathrm{G}$ (refer to Fig. 10). All the $\mathrm{Cu}$ stripes were independent, or not connected electrically. For the sake of simplicity, a square-type stripe $b_{x}=b_{y}=2.5 \mathrm{~mm}$ was chosen. The conductivity of each stripe $\sigma$ was given by $5.9 \times 10^{7} / \Omega \mathrm{m}$. There were a total $44 \mathrm{Cu}$ stripes (two of them at $x=0$ ) in order to reduce the necessary memory size.

The accuracy of the simulated results will now be discussed by changing the mesh size along the time axis. Figure 11 gives the field modulation at the center of the chamber for the different size of mesh. The red, blue, and black points describe the results where the mesh size was

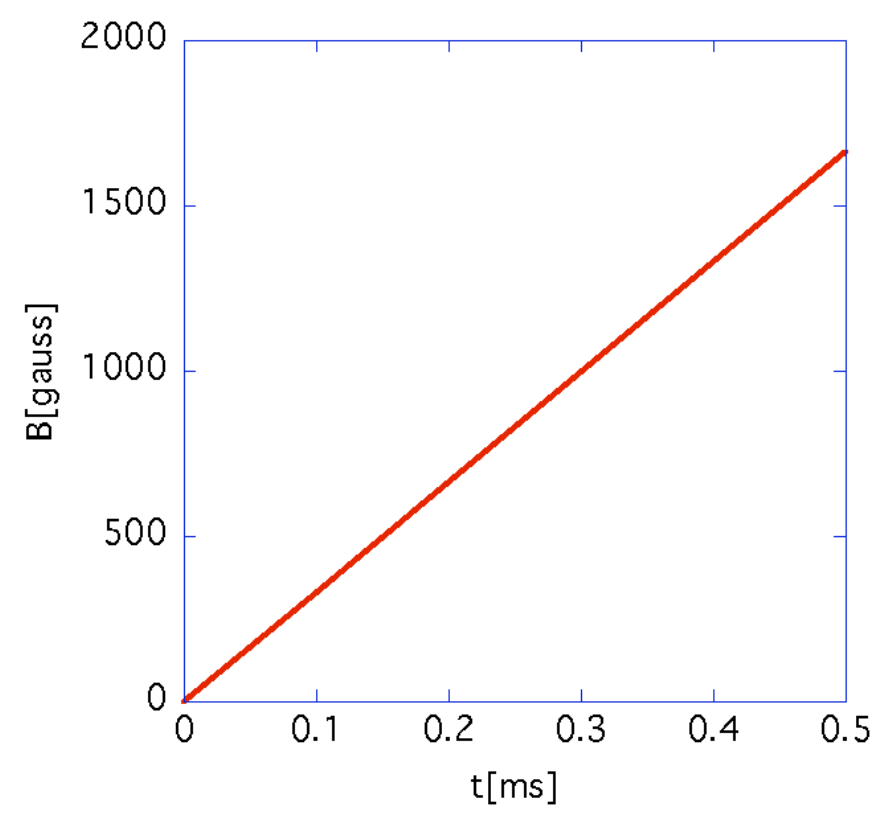

FIG. 10. (Color) The magnetic field is excited during $0.5 \mathrm{~ms}$ and its flux density at $x=y=0$ to have become $1664 \mathrm{G}$ (analytical data). 


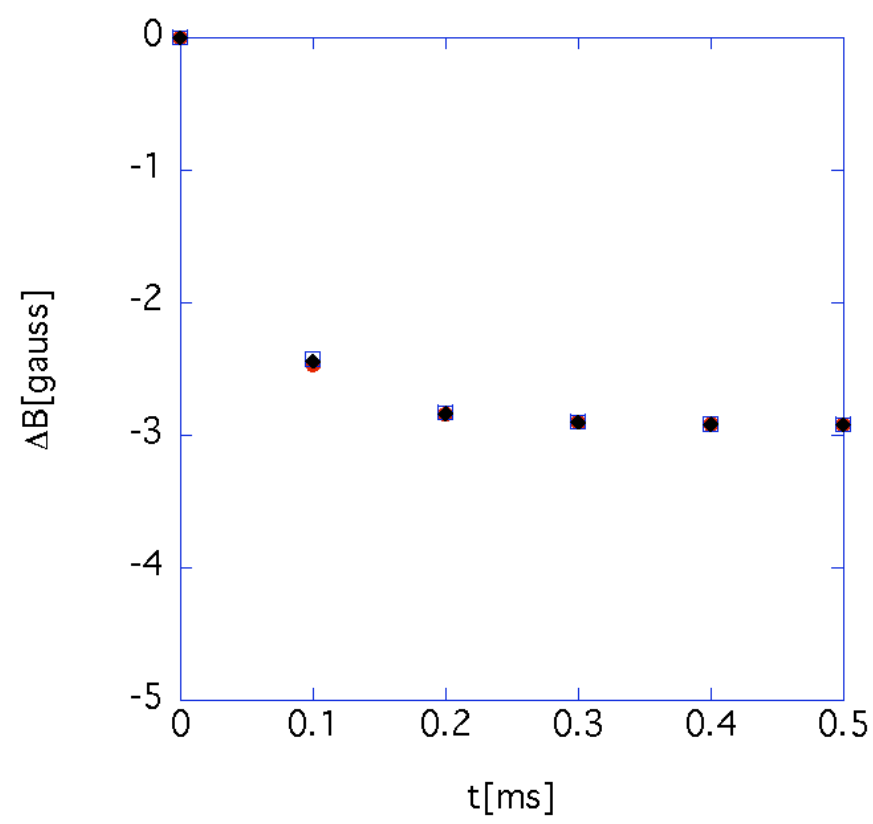

FIG. 11. (Color) The field modulation at the center of the chamber. The red, blue, and black points describe the results where the mesh size was $0.1,0.05$, and $0.025 \mathrm{~ms}$, respectively.

$0.1 \mathrm{~ms}, 0.05 \mathrm{~ms}$, and $0.025 \mathrm{~ms}$, respectively. A $0.025 \mathrm{~ms}$ mesh size proved sufficiently small to obtain the correct results, as they were sufficiently convergent.

In preparing to compare the simulated results with the theoretical results, it was necessary to confirm that the condition given in Eq. (6) was satisfied $(0.5 \mathrm{~ms}$ could be

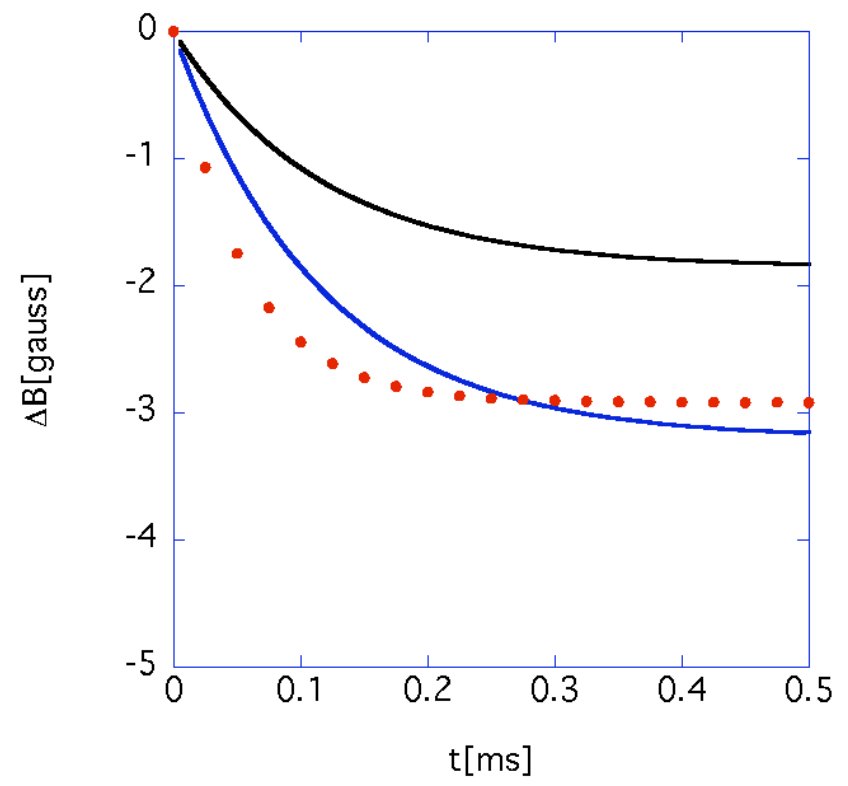

selected as time $T_{0}$ ), because it is indispensable in deriving Eq. (13). In fact, it could be satisfied as follows:

$$
\frac{Z_{0} \sigma b^{2}}{4 c T_{0}} \sim 0.23<1
$$

The simulated results will now be compared with the theoretical results. Both the simulated and theoretical results are given in Fig. 12. The left figure denotes the field modulation at the center of the chamber while the right figure gives the maximum current density [i.e. the current density $i(\xi, \eta)$ at the edge of stripe $\xi=b_{x}$ ]. The red points give the simulated results while the blue line gives the theoretical results. The black line in the left figure represents the results of the case where the shape of the stripe is cylindrical $(b=2.5 \mathrm{~mm})$. The simulated and theoretical results are in good agreement for the amount of field modulation and current density. A small discrepancy however does appear in the excitation time. This is a result of the approximation that the radius of cylindrical stripe $b$ could be replaced by the horizontal half size of rectangular stripe $b_{x}$ in deriving the formula for the field modulation of Eq. (18). Comparing the results where the shape of the stripe is square (blue line) with where the shape of the stripe is cylindrical (black line), as in the left of Fig. 12, it can be seen that the field modulation with the cylindrical stripe is about $2 / 3$ smaller than that with the rectangular stripe. This drop in field modulation can be explained by the fact that the cross section of the cylindrical stripe is smaller than that of the square type.

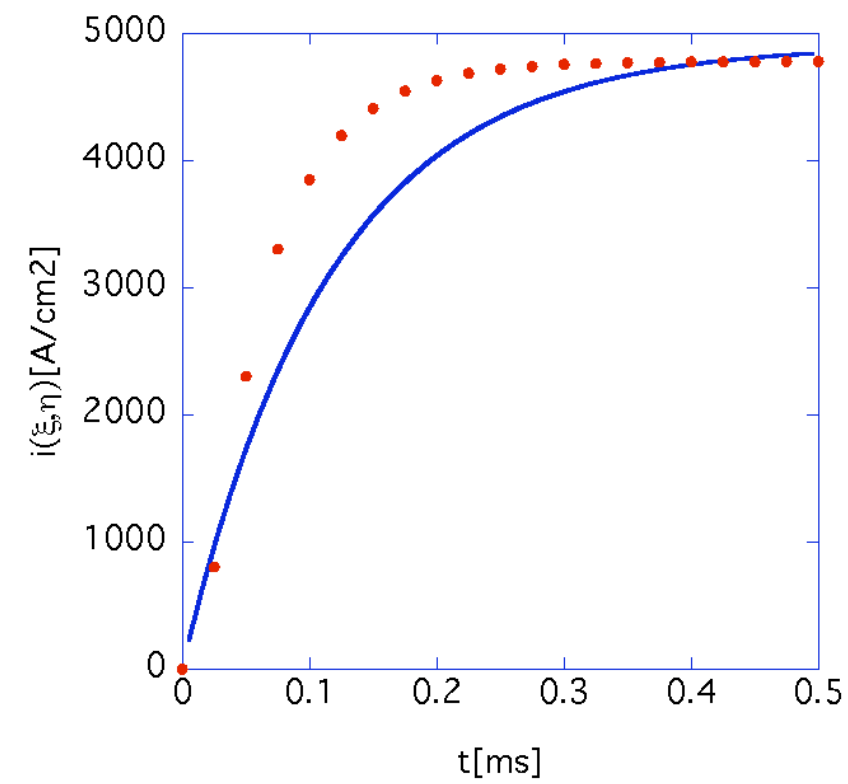

FIG. 12. (Color) The field modulation at the center of the chamber (the left figure) and the current density at the edge of stripe (the right figure). This field modulation is caused by the magnetic flux traversing isolated $\mathrm{Cu}$ stripes. The chamber radius $a$ is $15.25 \mathrm{~cm}$ and its longitudinal length is $80.0 \mathrm{~cm}$. The gap height $2 \mathrm{~g}$ is $32.0 \mathrm{~cm}$ and the gap width $2 h$ is $64.0 \mathrm{~cm}$. The red points describe the simulated (numerical) results and the blue line does the theoretical (analytical) results where the stripe is square: $5 \mathrm{~mm} \times 5 \mathrm{~mm}$. The black line in the left figure represents the theoretical (analytical) results where the stripe is cylindrical: $b=2.5 \mathrm{~mm}$. 
Since the theory has proved to be capable of providing sufficiently accurate results for the field modulation, the influence on the beam of the magnetic flux $B(t)$ that makes the bump orbit during the injection period is now considered. As a simple model, this can be described using

$$
\begin{aligned}
B(t)= & D\left(t-t_{0}\right) \Theta\left(t-t_{0}\right)-D\left(t-t_{0}\right) \Theta\left(t-t_{1}\right) \\
& +D\left(t_{1}-t_{0}\right) \Theta\left(t-t_{1}\right) \\
& -\frac{D\left(t_{1}-t_{0}\right)}{t_{3}-t_{2}}\left(t-t_{2}\right) \Theta\left(t-t_{2}\right) \\
& +\frac{D\left(t_{1}-t_{0}\right)}{t_{3}-t_{2}}\left(t-t_{3}\right) \Theta\left(t-t_{3}\right),
\end{aligned}
$$

$\Theta(t)$ as the step function and $D$ the constant value. Magnetic flux $B(t)$ is characterized by the rising time $t_{1}-$ $t_{0}$, flattop time $t_{2}-t_{1}$, and damping time $t_{3}-t_{2}$, as given in Fig. 13. For the sake of simplicity, only the case where the shape of the stripe is cylindrical is considered. Substituting the first differential of Eq. (28) with time into Eq. (13), the field modulation can finally be expressed as

$$
\begin{array}{rlrl}
\Delta B_{y, \text { ferr }}= & \frac{D Z_{0} b^{4} \sigma}{8 c}\left(1-e^{\left[(4 c) /\left(Z_{0} b^{2} \sigma\right)\right]\left(t_{0}-t\right)}\right) \sum_{i=1}^{N} \sum_{m, n=-\infty}^{\infty}(-1)^{m} \frac{\left[2 m h+(-1)^{m} x_{i}\right]^{2}-\left[2 n g+(-1)^{n} y_{i}\right]^{2}}{\left\{\left[2 m h+(-1)^{m} x_{i}\right]^{2}+\left[2 n g+(-1)^{n} y_{i}\right]^{2}\right\}^{2}} & \text { for } t_{0}<t<t_{1}, \\
\Delta B_{y, \text { ferr }}= & \frac{D Z_{0} b^{4} \sigma}{8 c}\left(e^{\left[(4 c) /\left(Z_{0} b^{2} \sigma\right)\right]\left(t_{1}-t\right)}-e^{\left[(4 c) /\left(Z_{0} b^{2} \sigma\right)\right]\left(t_{0}-t\right)}\right) & \\
& \times \sum_{i=1}^{N} \sum_{m, n=-\infty}^{\infty}(-1)^{m} \frac{\left[2 m h+(-1)^{m} x_{i}\right]^{2}-\left[2 n g+(-1)^{n} y_{i}\right]^{2}}{\left\{\left[2 m h+(-1)^{m} x_{i}\right]^{2}+\left[2 n g+(-1)^{n} y_{i}\right]^{2}\right\}^{2}} & & \text { for } t_{1}<t<t_{2}, \\
\Delta B_{y, \text { ferr }}= & \frac{D Z_{0} b^{4} \sigma}{8 c}\left[e^{\left[(4 c) /\left(Z_{0} b^{2} \sigma\right)\right]\left(t_{1}-t\right)}-e^{\left[(4 c) /\left(Z_{0} b^{2} \sigma\right)\right]\left(t_{0}-t\right)}-\frac{t_{1}-t_{0}}{t_{3}-t_{2}}\left(1-e^{\left[(4 c) /\left(Z_{0} b^{2} \sigma\right)\right]\left(t_{2}-t\right)}\right)\right] & & \text { for } t_{2}<t<t_{3}, \\
& \times \sum_{i=1}^{N} \sum_{m, n=-\infty}^{\infty}(-1)^{m} \frac{\left[2 m h+(-1)^{m} x_{i}\right]^{2}-\left[2 n g+(-1)^{n} y_{i}\right]^{2}}{\left\{\left[2 m h+(-1)^{m} x_{i}\right]^{2}+\left[2 n g+(-1)^{n} y_{i}\right]^{2}\right\}^{2}} & \\
\Delta B_{y, \text { ferr }}= & \frac{D Z_{0} b^{4} \sigma}{8 c}\left[e^{\left[(4 c) /\left(Z_{0} b^{2} \sigma\right)\right]\left(t_{1}-t\right)}-e^{\left[(4 c) /\left(Z_{0} b^{2} \sigma\right)\right]\left(t_{0}-t\right)}-\frac{t_{1}-t_{0}}{t_{3}-t_{2}}\left(e^{\left[(4 c) /\left(Z_{0} b^{2} \sigma\right)\right]\left(t_{3}-t\right)}-e^{\left[(4 c) /\left(Z_{0} b^{2} \sigma\right)\right]\left(t_{2}-t\right)}\right)\right] \\
& \times \sum_{i=1}^{N} \sum_{m, n=-\infty}^{\infty}(-1)^{m} \frac{\left[2 m h+(-1)^{m} x_{i}\right]^{2}-\left[2 n g+(-1)^{n} y_{i}\right]^{2}}{\left\{\left[2 m h+(-1)^{m} x_{i}\right]^{2}+\left[2 n g+(-1)^{n} y_{i}\right]^{2}\right\}^{2}} & \text { for } t_{3}<t .
\end{array}
$$

In order to clarify the field modulation property caused by the eddy current, the chamber is surrounded by the magnet yoke with an equal gap height and gap width of $2 g=2 h$. The magnetic flux is assumed to be excited as given in Fig. 13 [characterized by the rising time $t_{1}(=-800 \mu \mathrm{s})-t_{0}(=-300 \mu \mathrm{s}), \quad$ flattop time $t_{2}(=300 \mu \mathrm{s})-t_{1}(=-300 \mu \mathrm{s})$, and damping time $\left.t_{3}(=800 \mu \mathrm{s})-t_{2}(=300 \mu \mathrm{s})\right]$. The same parameters were used for the chamber as those in the previous case. Namely, the longitudinal length of chamber $l=80.0 \mathrm{~cm}$, its radius $a=15.25 \mathrm{~cm}$, and the radius of each $\mathrm{Cu}$ stripe $b$ $2.5 \mathrm{~mm}$. The number of $\mathrm{Cu}$ stripes $N$ was especially chosen to be 100 in order to obtain a more realistic field modulation than that in the previous case.

Let us imagine a situation where the chamber is covered in stripes from the lower side; for example, five cases where 20,40,60,80, and 100 stripes covering the chamber can be used. The case where the chamber is not surrounded by the magnet yoke is initially considered, because image currents do not have to be considered. The results are given 

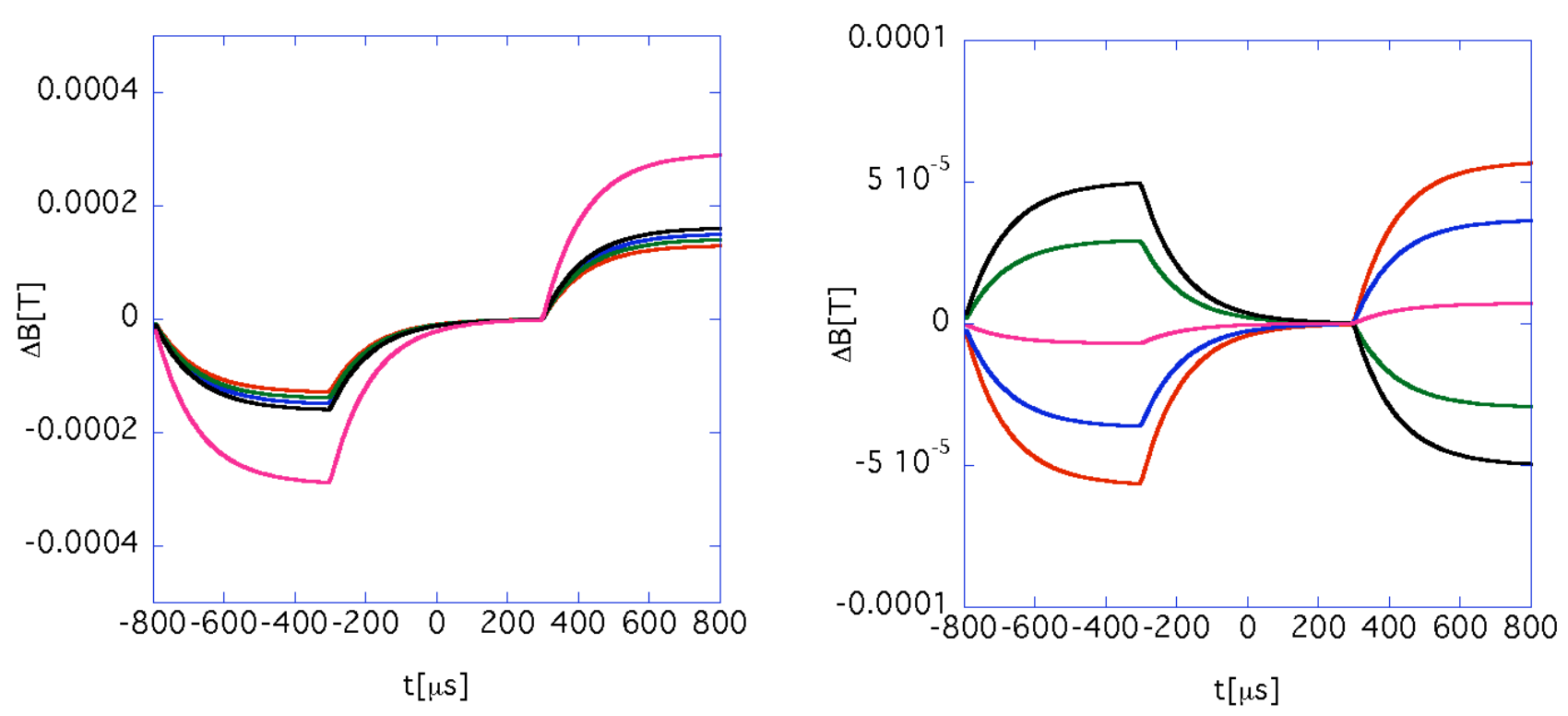

FIG. 14. (Color) The dependence of the field modulation at the center of the chamber on the number of $\mathrm{Cu}$ stripes, when the magnetic flux traverses the $\mathrm{Cu}$ stripes themselves. As a typical set of parameters, the radius of the chamber $a=15.25 \mathrm{~cm}$ and the radius of $\mathrm{Cu}$ stripe $b$ is $2.5 \mathrm{~mm}$. The original magnetic flux changes as in Fig. 13 (analytical data). The right figure shows the theoretical results without the magnet yoke and the left figure does the results where the size of magnet yoke is specified as $2 h=2 \mathrm{~g}=32.0 \mathrm{~cm}$. The red, blue, green, black, and pink lines represent the cases that 20,40,60,80, and 100 stripes cover the chamber from the lower side. The 100 stripes corresponds to the case where the chamber is completely covered by $\mathrm{Cu}$ stripes. The field modulation might become the smallest when the chamber is completely or half covered by stripes when the effect of the yoke could be negligible, while it is the largest in reality in the case where the chamber is completely covered by the stripes due to "the effect of the yoke."

on the right in Fig. 14. The red, blue, green, black, and pink lines in this figure represent the results for the cases with $20,40,60,80$, and 100 stripes, respectively. It is possible to cancel the effect of the eddy current at the center of the chamber by summation of the effects of the stripes, particularly when the number of strips is 50 and 100 , because the dominant term is proportional to $\cos 2 \theta_{i}$ as given in Eq. (12).

On the other hand, the magnet yoke is very close to the chamber in reality. The left in Fig. 14 gives the results where the size of the magnet yoke was specified as $2 h=$ $2 g=32.0 \mathrm{~cm}$. The red, blue, green, black, and pink lines in this figure represent the results for the cases with 20,40, 60,80 , and 100 stripes, respectively. The situation is quite different from that without the yoke. In particular, the maximum field modulation is seen in the case where the chamber is completely surrounded by $\mathrm{Cu}$ stripes. Figure 15 gives the dependence of the field modulation on the size of the magnet yoke when the chamber is completely surrounded by $\mathrm{Cu}$ stripes $(N=100)$. The discovery was made that the effect of the yoke is negligible, when the size of the magnet yoke is sufficiently large (typically 3 times larger than the radius of the chamber $a$ ). However in reality, the effect is actually very significant.

The case where the magnetic flux $B(t)$ is provided with a more realistic field pattern will now be considered. The left in Fig. 16 is the magnetic flux used during the injection period with the $3 \mathrm{GeV}$ RCS at J-PARC. The original

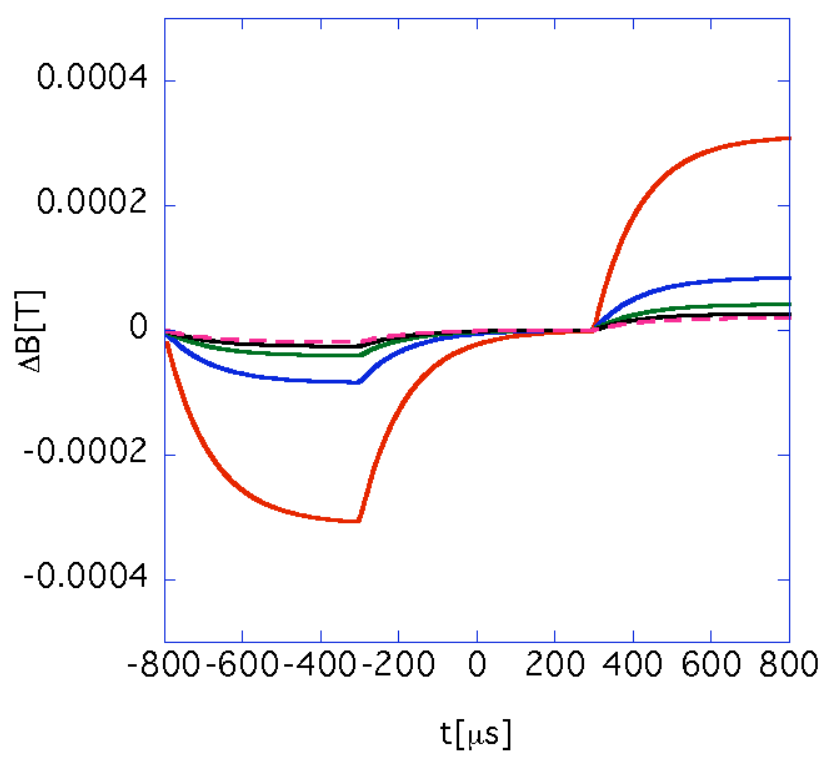

FIG. 15. (Color) The dependence of the field modulation (theoretical results) on the size of magnet yoke where the chamber is completely surrounded by $\mathrm{Cu}$ stripes $(N=100)$. The red, blue, green, black, and pink dotted lines represent the cases where both the gap height $g$ and the width $h$ is equal to $a$ (the radius of the chamber) $+b$ (the radius of the $\mathrm{Cu}$ stripe), where both $g$ and $h$ are 2 times larger than $a$, where both $g$ and $h$ are 3 times larger than $a$, where both $g$ and $h$ are 4 times larger than $a$ and where both $g$ and $h$ are 5 times larger than $a$, respectively. 

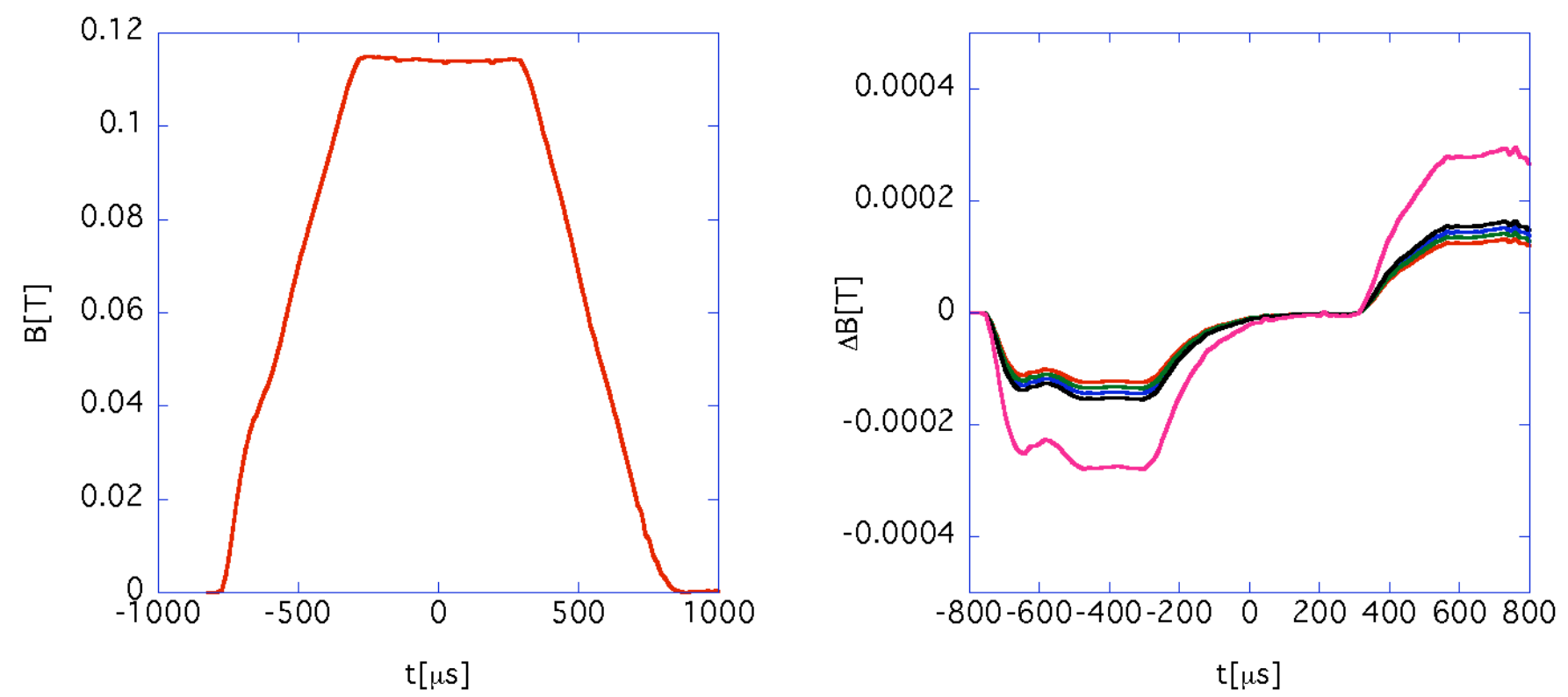

FIG. 16. (Color) The left figure represents the magnetic field pattern, which is used at the injection period with the $3 \mathrm{GeV}$ RCS at $\mathrm{J}$ PARC (measured data). The right figure represents the field modulation (theoretical results) at the center of the chamber when this (measured) magnetic flux traverses the $\mathrm{Cu}$ stripe. The red, blue, green, black, and pink lines represent the cases where 20, 40, 60, 80, and 100 stripes cover the chamber from the lower side.

measured field pattern was given for 500 ns intervals. However, the magnet power supply is provided with a low-pass filter at the output, the cutoff frequency of which is $70 \mathrm{kHz}$. The field data is then averaged over every $14 \mu \mathrm{s}$ to obtain that given on the left of Fig. 16 [16].

The theorem of partial integration enables the formula for the field modulation of Eq. (13) to be rewritten as follows:

$$
\begin{aligned}
\Delta B_{y, \text { ferr }}= & \left(B(t)-\frac{4 c}{Z_{0} b^{2} \sigma} \int_{-\infty}^{t} d t^{\prime} B\left(t^{\prime}\right) e^{\left[(4 c) /\left(Z_{0} b^{2} \sigma\right)\right]\left(t^{\prime}-t\right)}\right) \\
& \times \frac{b^{2}}{2} \sum_{i=1}^{N} \sum_{m, n=-\infty}^{\infty}(-1)^{m} \\
& \times \frac{\left[2 m h+(-1)^{m} x_{i}\right]^{2}-\left[2 n g+(-1)^{n} y_{i}\right]^{2}}{\left\{\left[2 m h+(-1)^{m} x_{i}\right]^{2}+\left[2 n g+(-1)^{n} y_{i}\right]^{2}\right\}^{2}},
\end{aligned}
$$

where the assumption is made that $B(t)=0$ at $t=-\infty$. Substituting this field pattern into Eq. (33) can be used to obtain the field modulation at the center of the chamber when the magnetic flux traverses the $\mathrm{Cu}$ stripe. The results are given on the right in Fig. 16. Comparing the results on the left of Fig. 14 with those on the right in Fig. 16 results in the discovery that the simple model, described using Eq. (28), is able to estimate the field modulation appropriately. The small discrepancy comes from the fact that the differential of the magnetic flux with time is not perfectly linear during the excitation and damping period.

\section{The field modulation due to the magnetic flux traversing the $\mathrm{Cu}$ coils}

Second, the field modulation due to the magnetic flux traversing the " $\mathrm{Cu}$ coils" is considered. Equation (25), or (26), describes the field modulation due to this effect. If the assumption is made that the bump magnet is excited in line with Eq. (28), the current on the $i$ th coil can be calculated by substituting the second differential of it with time into Eq. (24). The current on the $i$ th coil $I_{i}$ can then be obtained using

$I_{i}=0$

for $t<t_{0}$,

$I_{i}=-\sum_{j, k=1}^{N / 2} \frac{\operatorname{Dal} U_{i j} U_{j k}^{-1} \sin \theta_{k} e^{\left[\left(R / \lambda_{j}\right)+\left(1 / \lambda_{j}\right) \sqrt{R^{2}-\left(2 \lambda_{j} / C\right)}\right]\left(t_{0}-t\right)}}{\sqrt{R^{2}-\frac{2 \lambda_{j}}{C}}}+\sum_{j, k=1}^{N / 2} \frac{\operatorname{Dal} U_{i j} U_{j k}^{-1} \sin \theta_{k} e^{\left[\left(R / \lambda_{j}\right)-\left(1 / \lambda_{j}\right) \sqrt{R^{2}-\left(2 \lambda_{j} / C\right)}\right]\left(t_{0}-t\right)}}{\sqrt{R^{2}-\frac{2 \lambda_{j}}{C}}}$

for $t_{0}<t<t_{1}$, 


$$
\begin{aligned}
& I_{i}=-\sum_{j, k=1}^{N / 2} \frac{\operatorname{DalU}_{i j} U_{j k}^{-1} \sin \theta_{k}\left(e^{\left[\left(R / \lambda_{j}\right)+\left(1 / \lambda_{j}\right) \sqrt{R^{2}-\left(2 \lambda_{j} / C\right)}\right]\left(t_{0}-t\right)}-e^{\left[\left(R / \lambda_{j}\right)+\left(1 / \lambda_{j}\right) \sqrt{R^{2}-\left(2 \lambda_{j} / C\right)}\right]\left(t_{1}-t\right)}\right)}{\sqrt{R^{2}-\frac{2 \lambda_{j}}{C}}} \\
& +\sum_{j, k=1}^{N / 2} \frac{\operatorname{DalU}_{i j} U_{j k}^{-1} \sin \theta_{k}\left(e^{\left[\left(R / \lambda_{j}\right)-\left(1 / \lambda_{j}\right) \sqrt{R^{2}-\left(2 \lambda_{j} / C\right)}\right]\left(t_{0}-t\right)}-e^{\left[\left(R / \lambda_{j}\right)-\left(1 / \lambda_{j}\right) \sqrt{R^{2}-\left(2 \lambda_{j} / C\right)}\right]\left(t_{1}-t\right)}\right)}{\sqrt{R^{2}-\frac{2 \lambda_{j}}{C}}} \quad \text { for } t_{1}<t<t_{2} \text {, } \\
& I_{i}=-\sum_{j, k=1}^{N / 2} \frac{a l U_{i j} U_{j k}^{-1} \sin \theta_{k}}{\sqrt{R^{2}-\frac{2 \lambda_{j}}{C}}}\left[D\left(e^{\left[\left(R / \lambda_{j}\right)+\left(1 / \lambda_{j}\right) \sqrt{R^{2}-\left(2 \lambda_{j} / C\right)}\right]\left(t_{0}-t\right)}-e^{\left[\left(R / \lambda_{j}\right)+\left(1 / \lambda_{j}\right) \sqrt{R^{2}-\left(2 \lambda_{j} / C\right)}\right]\left(t_{1}-t\right)}\right)\right. \\
& \left.-D_{1} e^{\left[\left(R / \lambda_{j}\right)+\left(1 / \lambda_{j}\right) \sqrt{R^{2}-\left(2 \lambda_{j} / C\right)}\right]\left(t_{2}-t\right)}\right]+\sum_{j, k=1}^{N / 2} \frac{a l U_{i j} U_{j k}^{-1} \sin \theta_{k}}{\sqrt{R^{2}-\frac{2 \lambda_{j}}{C}}}\left[D \left(e^{\left[\left(R / \lambda_{j}\right)-\left(1 / \lambda_{j}\right) \sqrt{R^{2}-\left(2 \lambda_{j} / C\right)}\right]\left(t_{0}-t\right)}\right.\right. \\
& \left.\left.-e^{\left[\left(R / \lambda_{j}\right)-\left(1 / \lambda_{j}\right) \sqrt{R^{2}-\left(2 \lambda_{j} / C\right)}\right]\left(t_{1}-t\right)}\right)-D_{1} e^{\left[\left(R / \lambda_{j}\right)-\left(1 / \lambda_{j}\right) \sqrt{R^{2}-\left(2 \lambda_{j} / C\right)}\right]\left(t_{2}-t\right)}\right] \\
& I_{i}=-\sum_{j, k=1}^{N / 2} \frac{a l U_{i j} U_{j k}^{-1} \sin \theta_{k}}{\sqrt{R^{2}-\frac{2 \lambda_{j}}{C}}}\left[D\left(e^{\left[\left(R / \lambda_{j}\right)+\left(1 / \lambda_{j}\right) \sqrt{R^{2}-\left(2 \lambda_{j} / C\right)}\right]\left(t_{0}-t\right)}-e^{\left[\left(R / \lambda_{j}\right)+\left(1 / \lambda_{j}\right) \sqrt{R^{2}-\left(2 \lambda_{j} / C\right)}\right]\left(t_{1}-t\right)}\right)\right. \\
& \left.-D_{1}\left(e^{\left[\left(R / \lambda_{j}\right)+\left(1 / \lambda_{j}\right) \sqrt{R^{2}-\left(2 \lambda_{j} / C\right)}\right]\left(t_{2}-t\right)}-e^{\left[\left(R / \lambda_{j}\right)+\left(1 / \lambda_{j}\right) \sqrt{R^{2}-\left(2 \lambda_{j} / C\right)}\right]\left(t_{3}-t\right)}\right)\right] \\
& +\sum_{j, k=1}^{N / 2} \frac{a l U_{i j} U_{j k}^{-1} \sin \theta_{k}}{\sqrt{R^{2}-\frac{2 \lambda_{j}}{C}}}\left[D\left(e^{\left[\left(R / \lambda_{j}\right)-\left(1 / \lambda_{j}\right) \sqrt{R^{2}-\left(2 \lambda_{j} / C\right)}\right]\left(t_{0}-t\right)}-e^{\left[\left(R / \lambda_{j}\right)-\left(1 / \lambda_{j}\right) \sqrt{R^{2}-\left(2 \lambda_{j} / C\right)}\right]\left(t_{1}-t\right)}\right)\right. \\
& \left.-D_{1}\left(e^{\left[\left(R / \lambda_{j}\right)-\left(1 / \lambda_{j}\right) \sqrt{R^{2}-\left(2 \lambda_{j} / C\right)}\right]\left(t_{2}-t\right)}-e^{\left[\left(R / \lambda_{j}\right)-\left(1 / \lambda_{j}\right) \sqrt{R^{2}-\left(2 \lambda_{j} / C\right)}\right]\left(t_{3}-t\right)}\right)\right] \quad \text { for } t_{3}<t
\end{aligned}
$$

where $D_{1}=D\left(t_{1}-t_{0}\right) /\left(t_{3}-t_{2}\right)$. Summing these currents over index $i$ from 1 to $N / 2$ gives the modulation of magnetic field inside the rf-shielded chamber.

The feature of the field modulation when the current flows in a single coil in an open space is easier to understand than with a realistic complicated rf-shielded chamber inside a magnetic yoke. This case is considered first as the simplest example. In this case expressing the currents using Eqs. (34)-(38) becomes much more simple. Since it is already known that a general expression for the magnetic field is given by Eq. (19), field modulation $B_{y}$ at the center of the chamber can be obtained using:

$$
B_{y}=0
$$$$
\text { for } t<t_{0} \text {, }
$$

$$
B_{y}=\frac{Z_{0} D l \sin ^{2} \theta_{\mathrm{ID}}\left(e^{\left[\left(R / L_{\mathrm{ID}}\right)+\left(1 / L_{\mathrm{ID}}\right) \sqrt{R^{2}-\left(2 L_{\mathrm{ID}} / C\right)}\right]\left(t_{0}-t\right)}-e^{\left[\left(R / L_{\mathrm{ID}}\right)-\left(1 / L_{\mathrm{ID}}\right) \sqrt{R^{2}-\left(2 L_{\mathrm{ID}} / C\right)}\right]\left(t_{0}-t\right)}\right)}{c \pi \sqrt{R^{2}-\frac{2 L_{\mathrm{ID}}}{C}}} \quad \text { for } t_{0}<t<t_{1},
$$

$$
\begin{aligned}
& B_{y}=\frac{Z_{0} D l \sin ^{2} \theta_{\mathrm{ID}}}{c \pi \sqrt{R^{2}-\frac{2 L_{\mathrm{ID}}}{C}}}\left(e^{\left[\left(R / L_{\mathrm{ID}}\right)+\left(1 / L_{\mathrm{ID}}\right) \sqrt{R^{2}-\left(2 L_{\mathrm{ID}} / C\right)}\right]\left(t_{0}-t\right)}-e^{\left[\left(R / L_{\mathrm{ID}}\right)+\left(1 / L_{\mathrm{ID}}\right) \sqrt{R^{2}-\left(2 L_{\mathrm{ID}} / C\right)}\right]\left(t_{1}-t\right)}\right. \\
& \left.-e^{\left[\left(R / L_{\mathrm{ID}}\right)-\left(1 / L_{\mathrm{ID}}\right) \sqrt{R^{2}-\left(2 L_{\mathrm{ID}} / C\right)}\right]\left(t_{0}-t\right)}+e^{\left[\left(R / L_{\mathrm{ID}}\right)-\left(1 / L_{\mathrm{ID}}\right) \sqrt{R^{2}-\left(2 L_{\mathrm{ID}} / C\right)}\right]\left(t_{1}-t\right)}\right) \\
& \text { for } t_{1}<t<t_{2}
\end{aligned}
$$

$$
\begin{aligned}
& B_{y}=\frac{Z_{0} l \sin ^{2} \theta_{\mathrm{ID}}}{c \pi \sqrt{R^{2}-\frac{2 L_{\mathrm{ID}}}{C}}}\left[D\left(e^{\left[\left(R / L_{\mathrm{ID}}\right)+\left(1 / L_{\mathrm{ID}}\right) \sqrt{R^{2}-\left(2 L_{\mathrm{ID}} / C\right)}\right]\left(t_{0}-t\right)}-e^{\left[\left(R / L_{\mathrm{ID}}\right)+\left(1 / L_{\mathrm{ID}}\right) \sqrt{R^{2}-\left(2 L_{\mathrm{ID}} / C\right)}\right]\left(t_{1}-t\right)}\right)\right. \\
& -D_{1} e^{\left[\left(R / L_{\mathrm{ID}}\right)+\left(1 / L_{\mathrm{ID}}\right) \sqrt{R^{2}-\left(2 L_{\mathrm{ID}} / C\right)}\right]\left(t_{2}-t\right)}-D\left(e^{\left[\left(R / L_{\mathrm{ID}}\right)-\left(1 / L_{\mathrm{ID}}\right) \sqrt{R^{2}-\left(2 L_{\mathrm{ID}} / C\right)}\right]\left(t_{0}-t\right)}+e^{\left[\left(R / L_{\mathrm{ID}}\right)-\left(1 / L_{\mathrm{ID}}\right) \sqrt{\left.R^{2}-\left(2 L_{\mathrm{ID}} / C\right)\right]\left(t_{1}-t\right)}\right)}\right. \\
& \left.+D_{1} e^{\left[\left(R / L_{\mathrm{ID}}\right)-\left(1 / L_{\mathrm{ID}}\right) \sqrt{R^{2}-\left(2 L_{\mathrm{ID}} / C\right)}\right]\left(t_{2}-t\right)}\right] \\
& \text { for } t_{2}<t<t_{3}
\end{aligned}
$$




$$
\begin{aligned}
& B_{y}=\frac{Z_{0} l \sin ^{2} \theta_{\mathrm{ID}}}{c \pi \sqrt{R^{2}-\frac{2 L_{\mathrm{ID}}}{C}}}\left[D\left(e^{\left[\left(R / L_{\mathrm{ID}}\right)+\left(1 / L_{\mathrm{ID}}\right) \sqrt{R^{2}-\left(2 L_{\mathrm{ID}} / C\right)}\right]\left(t_{0}-t\right)}-e^{\left[\left(R / L_{\mathrm{ID}}\right)+\left(1 / L_{\mathrm{ID}}\right) \sqrt{R^{2}-\left(2 L_{\mathrm{ID}} / C\right)}\right]\left(t_{1}-t\right)}\right)\right. \\
& -D_{1}\left(e^{\left[\left(R / L_{\mathrm{ID}}\right)+\left(1 / L_{\mathrm{ID}}\right) \sqrt{R^{2}-\left(2 L_{\mathrm{ID}} / C\right)}\right]\left(t_{2}-t\right)}-e^{\left[\left(R / L_{\mathrm{ID}}\right)+\left(1 / L_{\mathrm{ID}}\right) \sqrt{R^{2}-\left(2 L_{\mathrm{ID}} / C\right)}\right]\left(t_{3}-t\right)}\right)-D\left(e^{\left[\left(R / L_{\mathrm{ID}}\right)-\left(1 / L_{\mathrm{ID}}\right) \sqrt{\left.R^{2}-\left(2 L_{\mathrm{ID}} / C\right)\right]\left(t_{0}-t\right)}\right.}\right. \\
& \left.\left.-e^{\left[\left(R / L_{\mathrm{ID}}\right)-\left(1 / L_{\mathrm{ID}}\right) \sqrt{R^{2}-\left(2 L_{\mathrm{ID}} / C\right)}\right]\left(t_{1}-t\right)}\right)+D_{1}\left(e^{\left[\left(R / L_{\mathrm{ID}}\right)-\left(1 / L_{\mathrm{ID}}\right) \sqrt{R^{2}-\left(2 L_{\mathrm{ID}} / C\right)}\right]\left(t_{2}-t\right)}-e^{\left[\left(R / L_{\mathrm{ID}}\right)-\left(1 / L_{\mathrm{ID}}\right) \sqrt{\left.R^{2}-\left(2 L_{\mathrm{ID}} / C\right)\right]}\left(t_{3}-t\right)\right.}\right)\right] \\
& \text { for } t_{3}<t \text {. }
\end{aligned}
$$

The field modulation due to the current of the coil specified by index ID $=1$ is now considered. As a typical parameter, capacitance $C$ is chosen to be $0.3 \mu \mathrm{F}$. The other parameters are the same as those in the right of Fig. 14. The results are given in Fig. 17. The damping time of the field modulation $\tau_{2}$ can be characterized by

$$
\tau_{2}=\frac{L_{\mathrm{ID}}}{R}
$$

In this case, it is evaluated as $\tau_{2}=622 \mu \mathrm{s}$, which is then consistent with the results of Fig. 17.

As mentioned in the Introduction, the attached capacitors are usually chosen of low impedance for the circulating beam, and high impedance for the induced current with the frequency components of the outside time-dependent magnetic field. Differing from the bending magnet of an RCS, a bump magnet can be of an even higher frequency. The power spectrum (Fourier transformation of the field pattern) for the bump magnet described in Fig. 13 is represented in Fig. 18. There is some doubt however that

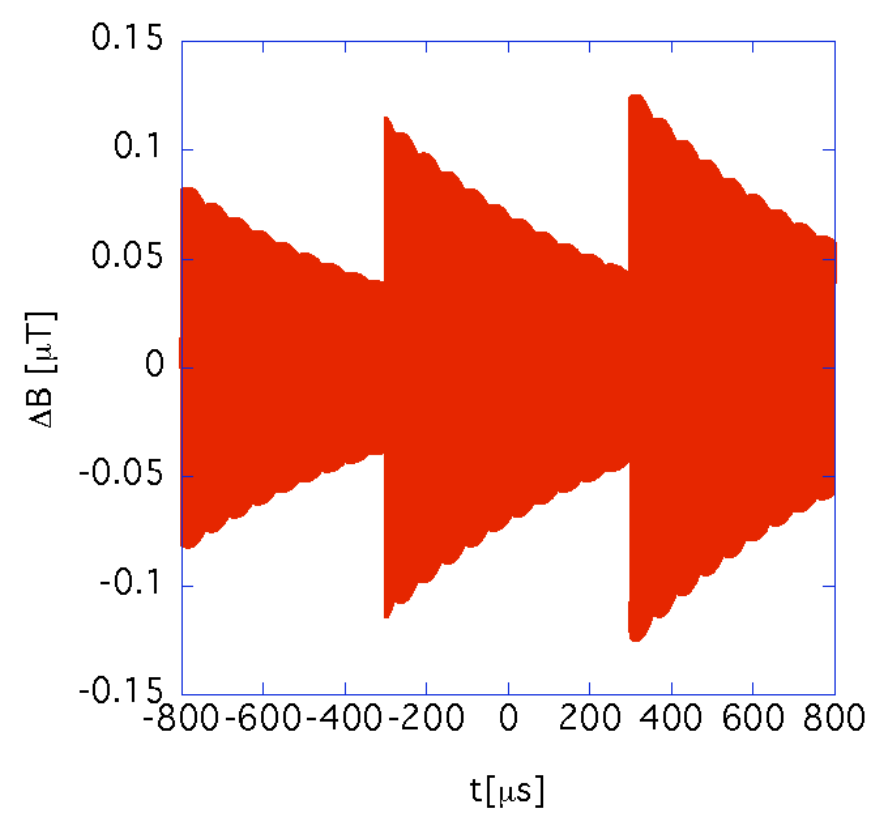

FIG. 17. (Color) The field modulation at the center of the chamber due to the current of only a single coil in an open space, which is specified with index $\mathrm{ID}=1$. As a typical parameter, capacitance $C=0.3 \mu \mathrm{F}$. The other parameters are the same as those in the right of Fig. 14. they show high impedance, for example, in the frequency peak around $1.5 \mathrm{kHz}$. The field modulation in the case where the coil in an open space has shorted ends is represented in Fig. 19. Comparing the results given in Fig. 17 with those in Fig. 19, it can be seen that the field modulation is enhanced by an approximate order of magnitude 3, because much more current is flowing in the stripes. This clarifies the role of capacitors as a blocking capacitor with the low frequency components.

Before proceeding with any further investigation it needs to be confirmed whether the simplification where the rf-shielded chamber with flanges is considered as a multicoil systems is appropriate or not. For this purpose, the induced current on the $\mathrm{Cu}$ stripes without any capacitors is simulated using OPERA-3D ELEKTRA. As represented in Fig. 20, the chamber is surrounded by a yoke whose longitudinal length is $80.0 \mathrm{~cm}$, and with gap height $2 g=$ $32.0 \mathrm{~cm}$ and gap width $2 h=64.0 \mathrm{~cm}$. The radius of chamber $a$ is $15.25 \mathrm{~cm}$. In order to also find the longitudinal leak effect of the original magnetic flux from the area where the magnet yoke is, the longitudinal length of chamber $l$ was considered to be twice as long as that of the yoke: namely $l=1.6 \mathrm{~m}$. Similar to the previous simulation that was

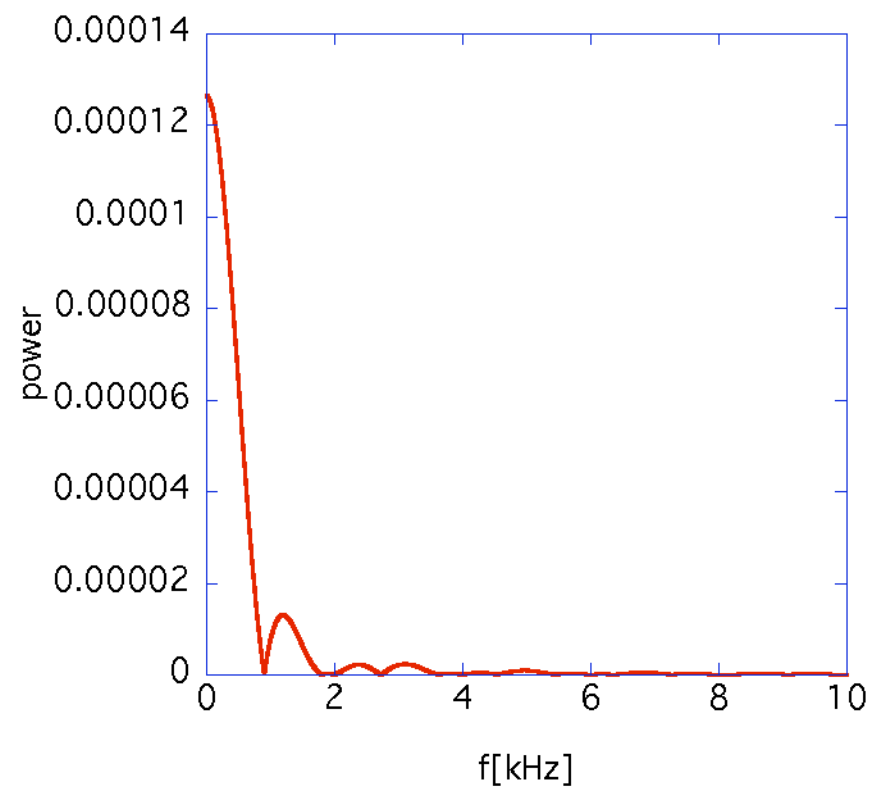

FIG. 18. (Color) Power spectrum for the field pattern $B(t)$ represented in Fig. 13. 


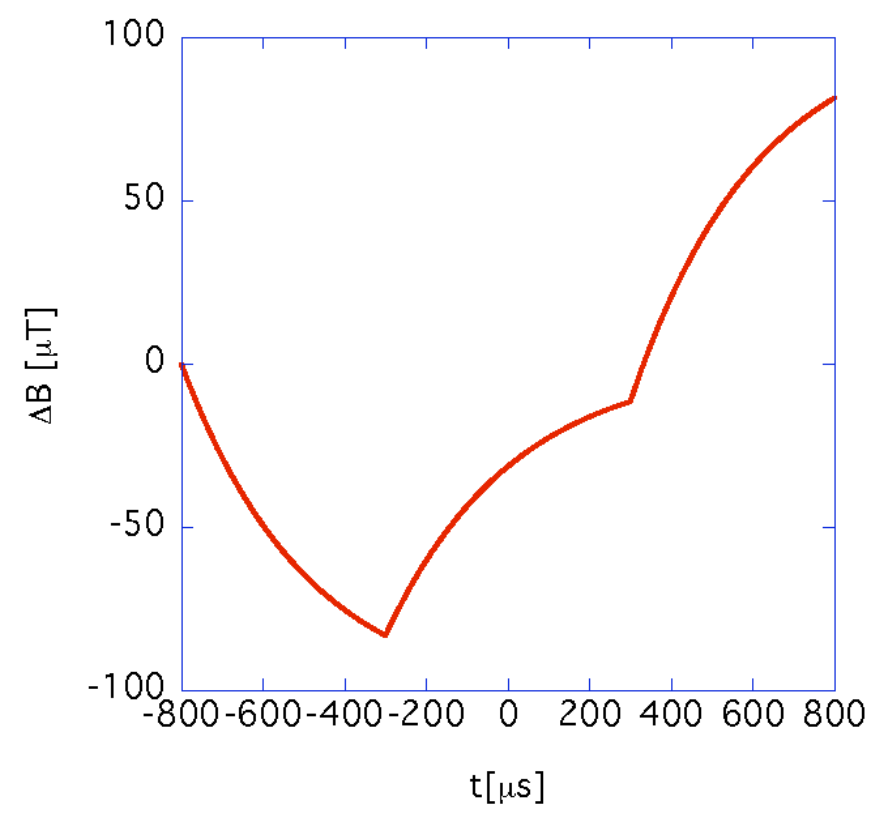

FIG. 19. (Color) The field modulation where the coil in an open space has shorted ends.

done to investigate the field modulation due to the magnetic flux traversing the $\mathrm{Cu}$ stripes, the magnetic field was assumed to have risen during $0.5 \mathrm{~ms}$ and that its flux density at $x=y=0$ would be $1664 \mathrm{G}$ (refer to Fig. 10). The total number of $\mathrm{Cu}$ stripes was 44 (two of them at $x=$ $0)$ so as to reduce the amount of memory needed. The

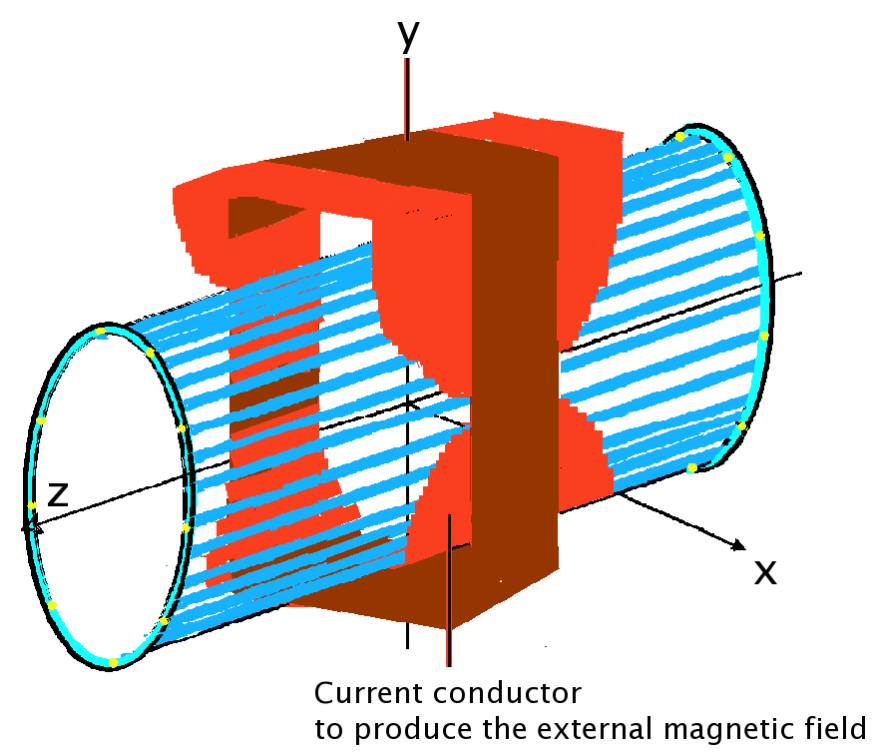

FIG. 20. (Color) The chamber that is surrounded by the ferromagnetic yoke with the current conductors whose longitudinal length is $80.0 \mathrm{~cm}$, gap height $2 \mathrm{~g}=32.0 \mathrm{~cm}$, and gap width $2 \mathrm{~h}=$ $64.0 \mathrm{~cm}$. The radius of chamber $a$ is $15.25 \mathrm{~cm}$. The longitudinal length of it is chosen to be $l=1.6 \mathrm{~m}$ which is twice as long as the yoke length to find the longitudinal leak effect of the original magnetic field from the area where the magnet yoke exists. shape of each stripe was a $5 \mathrm{~mm} \times 5 \mathrm{~mm}$ square. The resistance of each stripe was determined to be $10 \Omega$ in order that the induced magnetic field modulation would be a few percent when compared to the amount of the original magnetic field. This reduced conductivity makes the field modulation due to the magnetic flux traversing the $\mathrm{Cu}$ stripe about $0.01 \%$ smaller than that with case $\sigma=5.9 \times$ $10^{7} / \Omega \mathrm{m}$ [refer to Eqs. (29)-(32)]. That effect could then be ignored in this simulation.

The $\mathrm{Cu}$ stripes were specified as "ID", which was defined in Sec. II A (since the coils were defined by combining stripes that are symmetrical in this theory, $N=42$ needs to be substituted into the definition of $\theta_{\mathrm{ID}}$ ). Similar to the previous subsection, the accuracy of the results of this simulation was investigated by changing the mesh size along the time axis. Figure 21 gives the current density of the $\mathrm{Cu}$ stripes specified with ID $=11$, which corresponds to the largest coil in the system. The red, blue, and black points represent the case where the mesh size was $0.1,0.05$, and $0.025 \mathrm{~ms}$, respectively. The discovery was made that the $0.025 \mathrm{~ms}$ mesh size was sufficiently small to obtain the correct results similar to in the case discussed in the previous subsection.

The simulated results will now be compared with the theoretical results. Figure 22 gives the current density at $0.5 \mathrm{~ms}$ on the $\mathrm{Cu}$ stripes specified as ID, and the time dependence of the current density of the $\mathrm{Cu}$ stripes specified with an ID of 11. The red points represent the results of the simulation, while the blue points the theoretical results. In obtaining the above theoretical results, the assumption was that the uniform magnetic flux $B(t)$ would only tra-

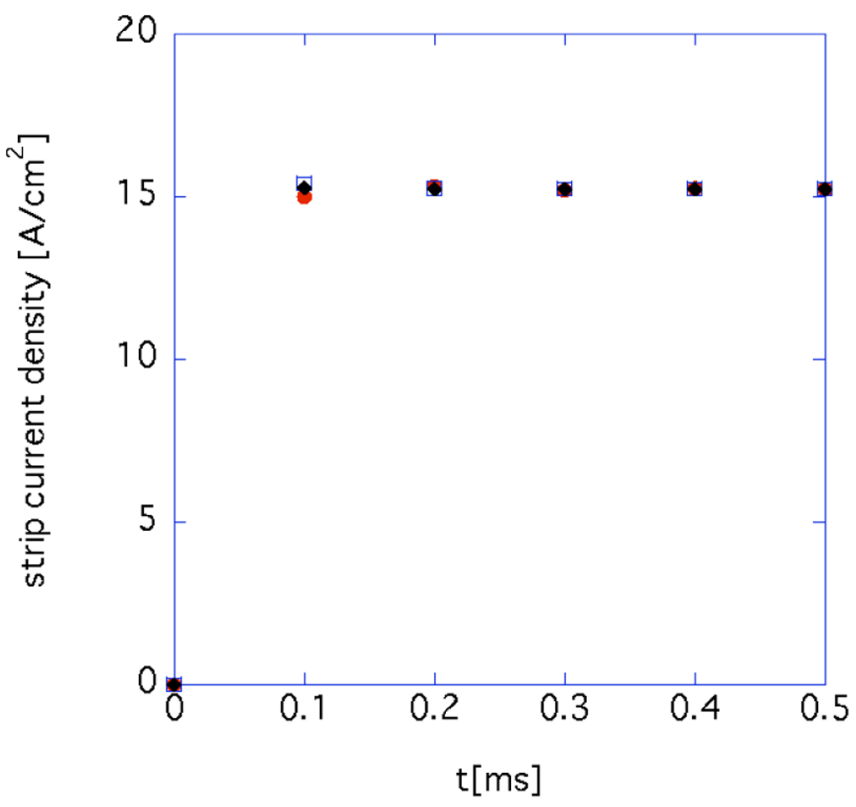

FIG. 21. (Color) The dependence on the mesh size of the current density for $\mathrm{Cu}$ stripes specified with ID $=11$. The red, blue, and black points represent the results where the mesh size is 0.1 , 0.05 , and $0.025 \mathrm{~ms}$, respectively. 

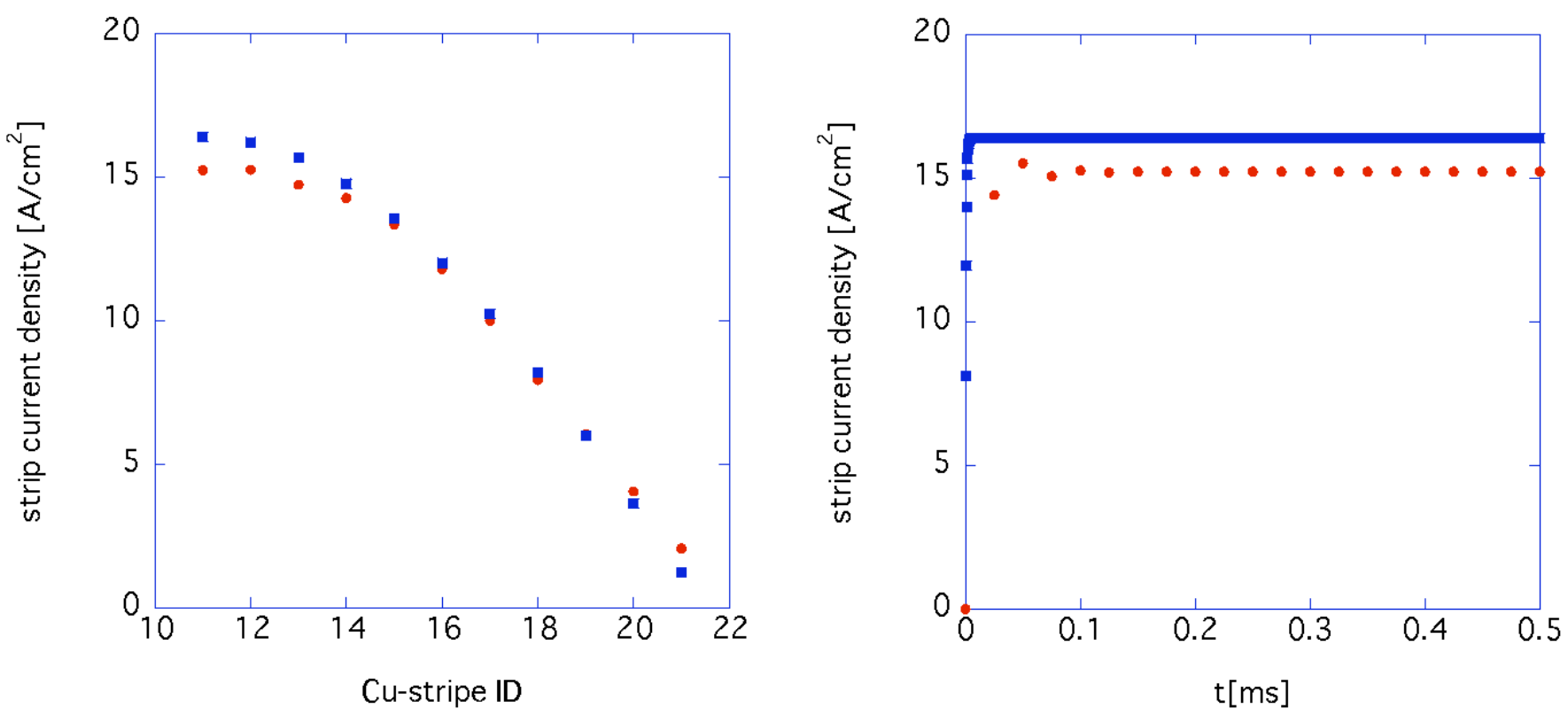

FIG. 22. (Color) Comparison between the theoretical and simulated results with OPERA-3D ELEKTRA where all the stripes have shorted ends. The left figure shows the current density at $0.5 \mathrm{~ms}$ on the $\mathrm{Cu}$ stripe specified with ID and the right figure does the time dependence of the current density for the $\mathrm{Cu}$ stripes specified with ID $=11$, which corresponds to the largest coil in this system. The red and blue points represent the simulated and theoretical results, respectively. The assumption was made in the theory that the uniform magnetic flux $B(t)$ traverses only the area specified by $-a \leq x \leq a$ and $-40 \mathrm{~cm} \leq z \leq 40 \mathrm{~cm}$. The theoretical results and the numerical calculation were in agreement with a below $6 \%-7 \%$ accuracy.

verse the area specified by $-a \leq x \leq a$ and $-40 \mathrm{~cm} \leq$ $z \leq 40 \mathrm{~cm}$ and that the inductance matrix $L_{i j}$ could be approximated using the summation of Eqs. (20)-(22) with $l=80 \mathrm{~cm}$. The theoretical results and the numerical calculation were in agreement with a below 6\%-7\% accuracy.

This discrepancy mainly arose from the assumption that the original magnetic field $B(t)$ was uniform and its longitudinal component zero. Actually, the current with ID = 11 was reestimated using information from the threedimensional $\vec{B}(t)$ used in the simulation as input in the theory. In this analysis, the original coil defined on the left of Fig. 6 had to be used, because the magnetic field has a longitudinal component. With this coil, magnetic flux $\Phi$ should be calculated as

$$
\Phi=\int_{\Omega} d S \vec{n} \cdot \vec{B}(t)
$$

where $\vec{n}$ is a normal vector perpendicular to the surface of the coil and integration region $\Omega$ is specified as the surface of each coil. This manipulation causes the left-hand side of Eq. (23) to need modifying in order for $B(t)$ to have the coil dependence of $B_{i}(t)$. In other words, magnetic flux density $D$ in Eqs. (34)-(38) should have the coil dependence of $D_{k}$. The result is given in Fig. 23. The agreement between the simulated and theoretical results is much better than the results depicted on the right in Fig. 22.

Since the validity of the theory had been confirmed, the rf-shielded chamber equipped with capacitors could be considered. From a realistic point of view, it is significant to consider the situation where the capacitors have been partially broken. If only $k$ capacitors are still working on the lower side of the chamber, the current can only flow on

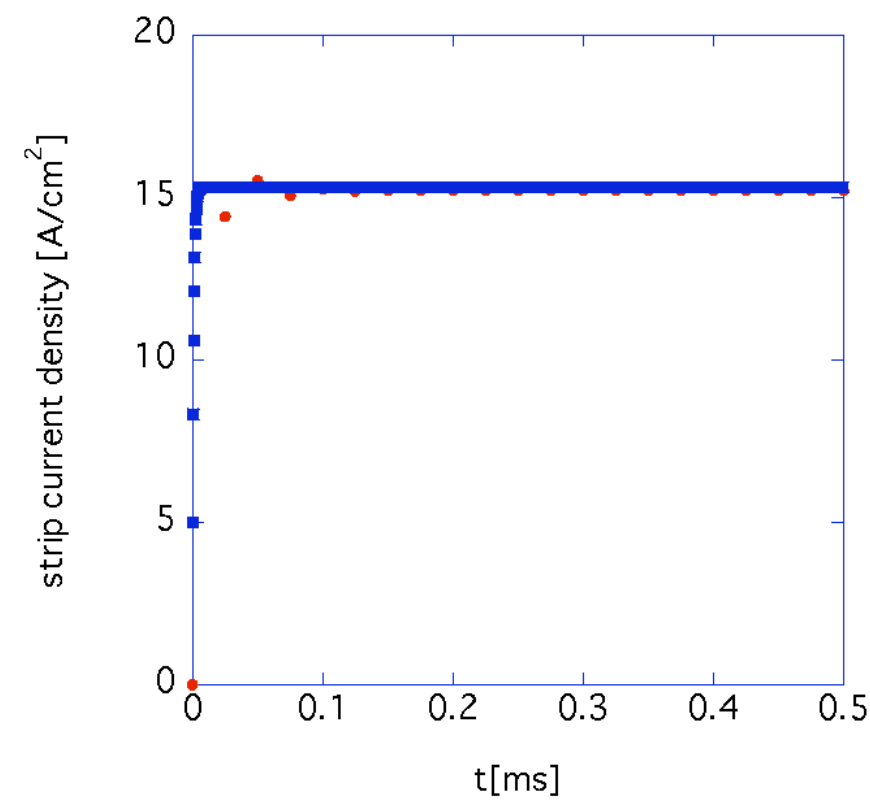

FIG. 23. (Color) The time dependence of the current density for the $\mathrm{Cu}$ stripes specified with ID $=11$. The red and blue points represent the simulated and theoretical results, respectively. Information from the three-dimensional $\vec{B}(t)$ is used in the simulation as input in the theory. The agreements of both results become much better than those on the right of Fig. 22. 
the $\mathrm{Cu}$ stripes. As a simple case, the situation where the original magnetic flux, as given in Fig. 13, was excited outside the chamber was considered. The dependence on the number of working capacitors $k$ of the field modulation is given in Fig. 24 (the capacitance $C=0.3 \mu \mathrm{F}$ was used as a typical parameter. The other parameters were the same as those in the left of Fig. 14). The left figure gives the behavior around $t=-300 \mu \mathrm{s}$, while the right figure behavior around $t=300 \mu \mathrm{s}$. The red, blue, and black lines represent the cases where the number of working capacitors $k$ were equal to 40,60 , and 100 , respectively. Since the initial condition was the same for all the cases, the larger the number of working capacitors $k$, the larger the amount of field modulation, for any time before $t_{1}$. After time $t_{1}$, the situation was not so simple. Since the eigenfrequencies of the coils differed in each case, the oscillation changes at time $t_{1}$ and $t_{2}$ had different timings. If the differential of the field modulation with time were to be positive at time $t_{1}$ or $t_{2}$, the oscillation would be enhanced, because the differential of the original magnetic field with time was reduced at those times. If the differential of it with time were to be negative, the oscillation would not have been enhanced when compared to the previous case. This phenomenon causes a reverse in the amount of field modulation with respect to the number of working capacitors. Actually, this reverse in the amount of field modulation can be seen in the

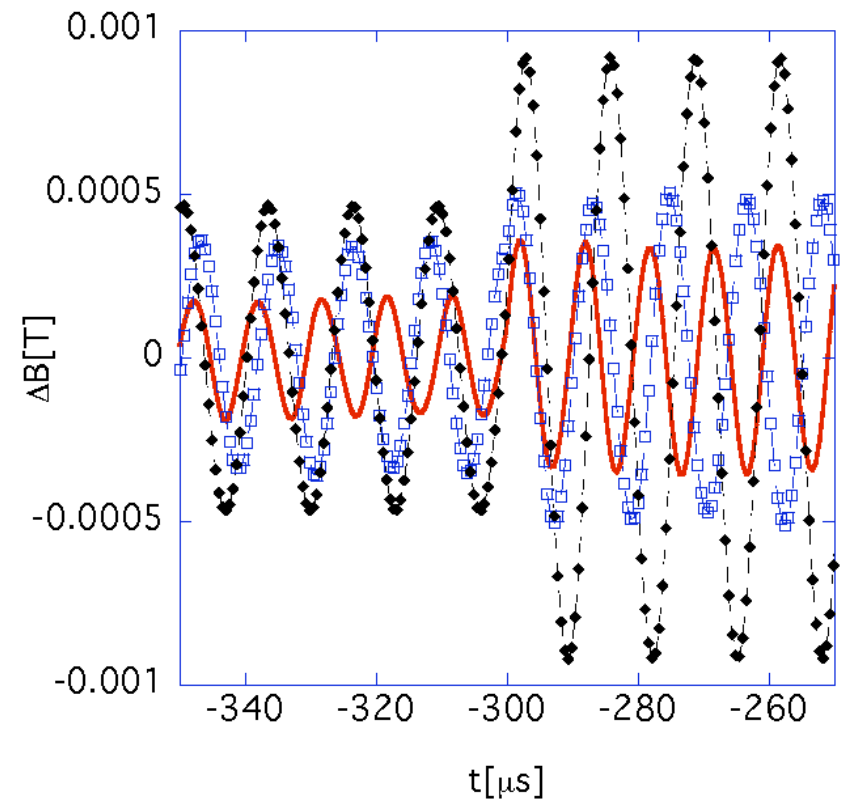

right of Fig. 24 under the field pattern given in Fig. 13. The field modulation for the case $k=60$ is smaller than that with the case $k=40$ for any time after $t=t_{2}=300 \mu \mathrm{s}$, although the number of working capacitors $k=60$ is bigger than $k=40$.

In order to use Eq. (25) or (26), the current on the $i$ th coil needs to be calculated correctly. If the mutual inductance between coils is ignored, the formula for the induced current on the $i$ th coil would be much simpler. Whether the effect of the mutual inductance could be ignored was investigated by comparing the results of Eqs. (34)-(38) with those without the effects of the mutual inductances. The results are given in Fig. 25. The left figure gives the case where 20 capacitors are working on the lower side of the chamber, while the right figure the case where all the capacitors $(k=100)$ are working. The red and blue points in those figures represent the results of Eqs. (34)-(38) and those using the formula where the mutual inductances were neglected, respectively. There are large discrepancies. The damping effect is reduced when the effect from the mutual inductances is included. This is because the $Q_{j}$ value ( $\equiv \lambda_{j} / R \sqrt{\lambda_{j} C}$ ) for the $j$ th mode is changed through the inductance matrix $L_{i j}$ being diagonalized, which is represented by Eqs. (20) and (21). Figure 26 gives the result of comparing the $Q_{j}$ value with and without the effect of the mutual inductance. Confirmation was made that the $Q_{j}$

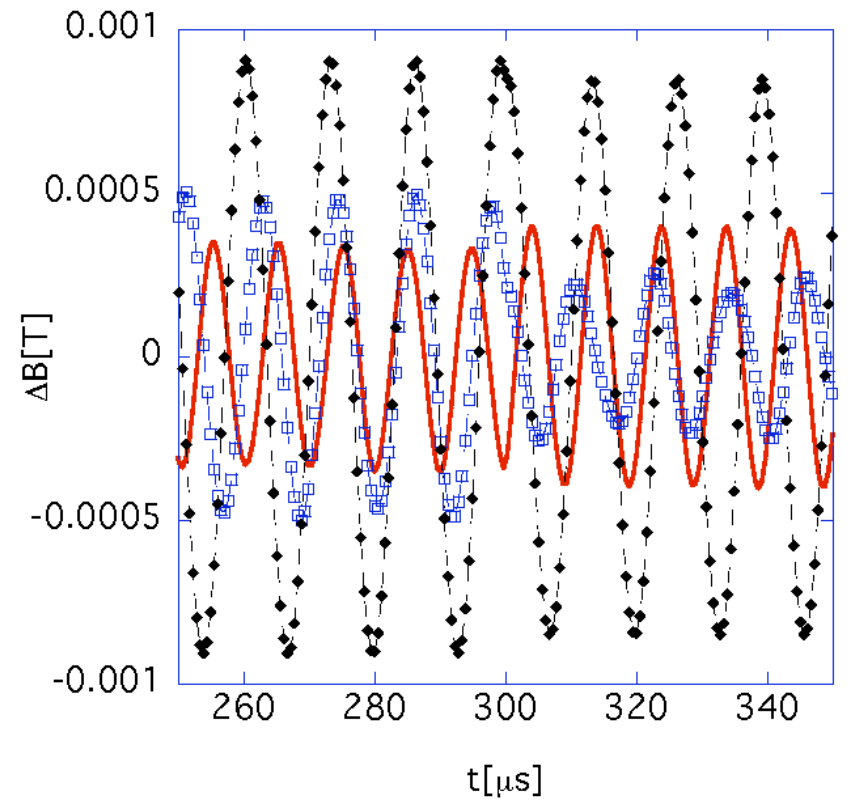

FIG. 24. (Color) The dependence on the number of working capacitors $k$ of the field modulation (theoretical results) caused by the magnetic flux traversing the $\mathrm{Cu}$ coils. The original magnetic flux is excited outside the chamber, as in Fig. 13 (analytical data). The left figure gives the behavior of field modulation around $t=-300 \mu \mathrm{s}$, while the right figure behavior around $t=300 \mu \mathrm{s}$. The red, blue, and black lines represent the cases where the number of working capacitors $k$ is 40,60 , and 100, respectively. The attached capacitance $C=0.3 \mu \mathrm{F}$ was used as a typical parameter, The other parameters are the same as in the left of Fig. 14. Namely, the chamber radius $a$ is $15.25 \mathrm{~cm}$, the radius of $\mathrm{Cu}$ stripe $b$ is $2.5 \mathrm{~mm}$, the total number of $\mathrm{Cu}$ stripes $N$ is 100 , and the conductivity of copper $\sigma$ is $5.9 \times 10^{7} / \Omega \mathrm{m}$. The chamber is surrounded by the magnet yoke with its longitudinal length $80 \mathrm{~cm}$, and its equal gap height $2 \mathrm{~g}=$ $32.0 \mathrm{~cm}$ and gap width $2 h=32.0 \mathrm{~cm}$. 

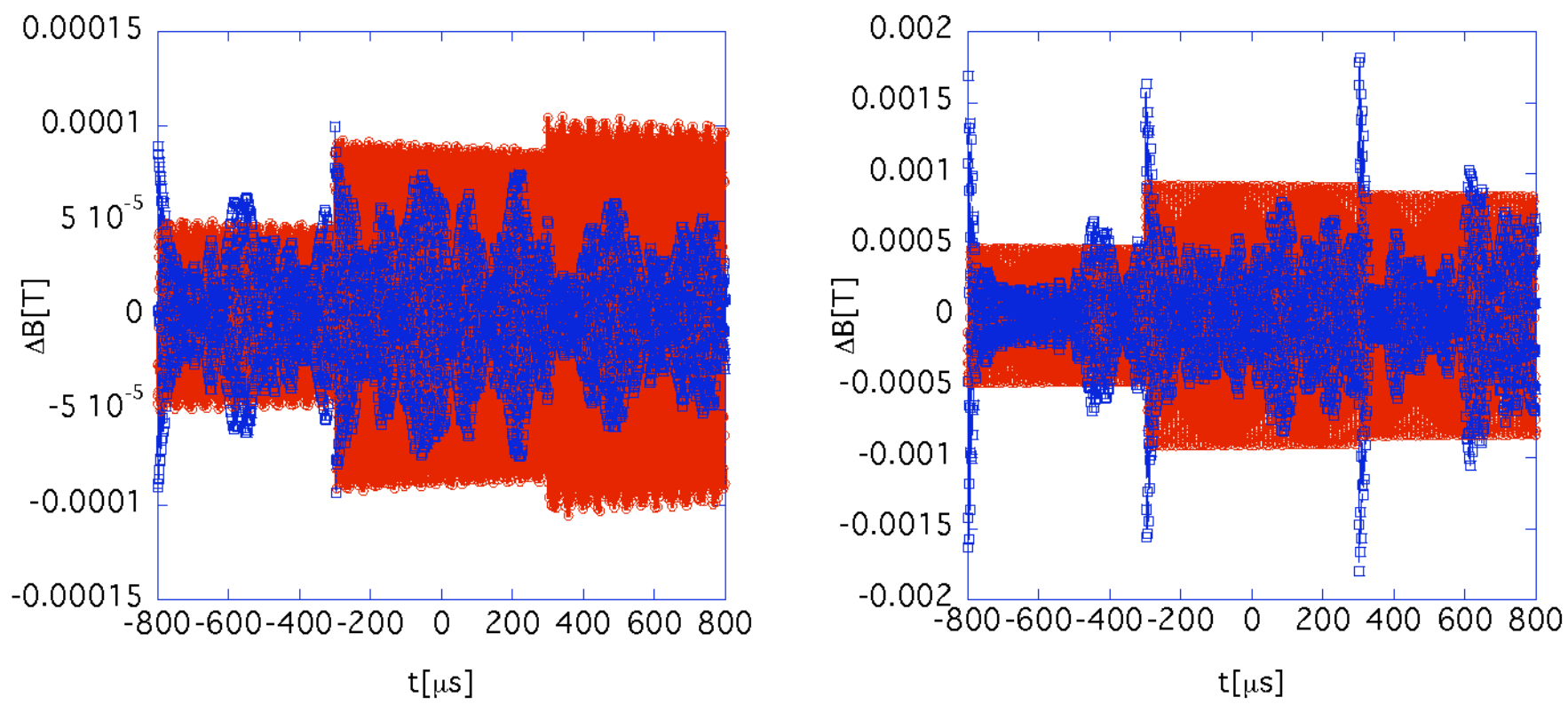

FIG. 25. (Color) The comparison of the field modulation (theoretical results) obtained by Eqs. (34)-(38) with that done by the formula where the mutual inductances are neglected. The left figure represents the case that 20 capacitors are working on the lower side of the chamber. The right figure represents the case where all the capacitors $(k=100)$, which cover the chamber, are working. The red points represent the results calculated by Eqs. (34)-(38) and blue points do those done by the formula where the mutual inductances are neglected. It is indispensable to consider the effect of mutual inductance, in order to evaluate the field modulation correctly.

value had indeed been enhanced by the diagonalization. In other words, it should be noticeable that the amount of field modulation had been enhanced as a whole, when compared to the case where only the effect from the self-inductance

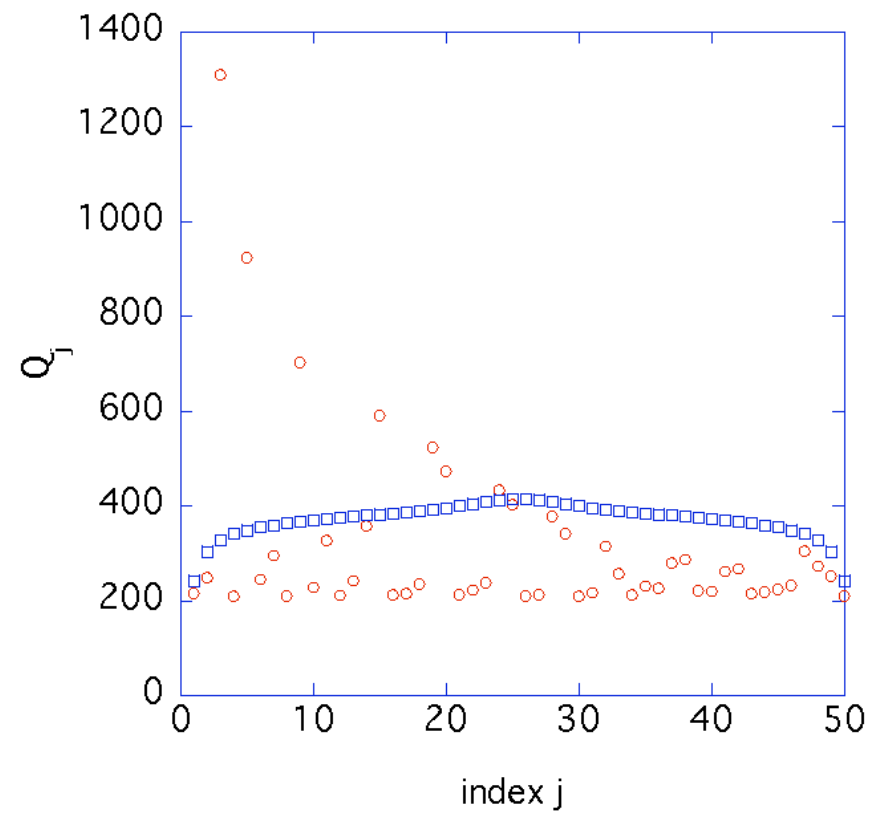

FIG. 26. (Color) The red and blue points represent $Q_{j}$ value with and without the effect of the mutual inductance, respectively. The $Q_{j}$ value for the $j$ th mode had been enhanced by the inductance matrix $L_{i j}$ being diagonalized, when compared to the case where the mutual inductance is neglected. was included, thus making it indispensable in evaluating the field modulation correctly that the effect of the mutual inductances between all the coils be considered. Once the current on the $i$ th coil is obtained, Eq. (25) or (26) can describe the field modulation inside the chamber quite appropriately.

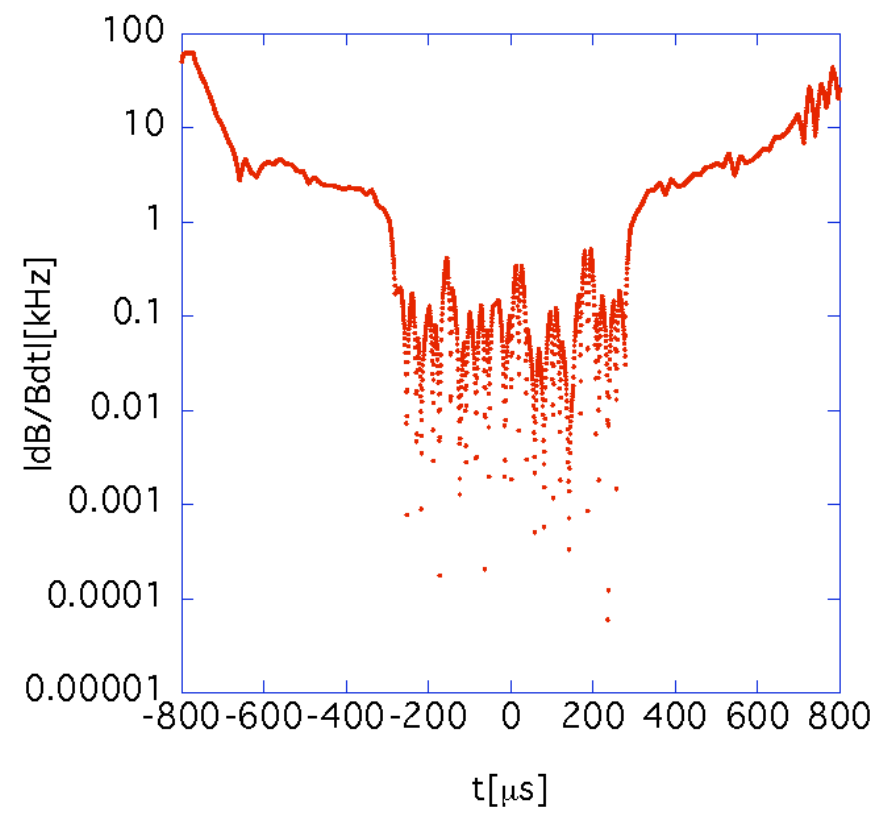

FIG. 27. (Color) The rate of change of magnetic flux $|d B / B d t|$ for the "measured" field pattern that is given by the left of Fig. 16. 
There is a possibility that the analysis using the above model, where the original magnetic flux follows the field pattern given in Fig. 13, has been too simplified to use in estimating the field modulation, because a derivative discontinuity exists with magnetic flux $B(t)$ in Eq. (24) when Eq. (28) is used as the field pattern. Similar to the case in the previous subsection, a more realistic field pattern should be used, like the left in Fig. 16. As this figure reveals, different to the pattern given in Fig. 13, the differential of the magnetic flux with time is not constant in the excitation and damping periods. The rate of change of magnetic flux $|d B / B d t|$, which is given in Fig. 27, provides more information on this field pattern. The amount of $|d B / B d t|$ can be comparable to the normal mode frequencies $1 / 2 \pi \sqrt{\lambda_{i} C}$ for this rf-shielded chamber (refer to Fig. 32). It can also be seen that the magnetic flux actually fluctuates even in the flattop period. This could mean that the traverse of the $\mathrm{Cu}$ coils by the magnetic flux following this field pattern induces the large field modulation inside the chamber.

When the derivative of magnetic flux $B(t)$ with time is continuous, the formula for the induced current described in Eq. (24) can be rewritten using the theorem of partial integration. The result is then expressed as follows:

$$
\begin{aligned}
I_{i}= & \sum_{j, k=1}^{N / 2} \frac{U_{i j} 2 B(t) a l U_{j k}^{-1} \sin \theta_{k}}{\lambda_{j}}-\sum_{j, k=1}^{N / 2} \frac{U_{i j}\left(5 R^{2}-\frac{2 \lambda_{j}}{C}\right) a l U_{j k}^{-1} \sin \theta_{k}}{\lambda_{j}^{2} \sqrt{\frac{2 \lambda_{j}}{C}-R^{2}}} \int_{-\infty}^{t} d t^{\prime} B\left(t^{\prime}\right) e^{\left(R / \lambda_{j}\right)\left(t^{\prime}-t\right)} \sin \frac{\sqrt{\frac{2 \lambda_{j}}{C}-R^{2}}}{2 \lambda_{j}}\left(t^{\prime}-t\right) \\
& -\sum_{j, k=1}^{N / 2} \frac{U_{i j} 4 R a l U_{j k}^{-1} \sin \theta_{k}}{\lambda_{j}^{2}} \int_{-\infty}^{t} d t^{\prime} B\left(t^{\prime}\right) e^{\left(R / \lambda_{j}\right)\left(t^{\prime}-t\right)} \cos \frac{\sqrt{\frac{2 \lambda_{j}}{C}-R^{2}}}{2 \lambda_{j}}\left(t^{\prime}-t\right),
\end{aligned}
$$

where the assumption is made that magnetic flux $B(t)$ and its derivative with time $d B(t) / d t$ at $t=-\infty$ is zero. After substituting the magnetic flux, which is shown in the left of Fig. 16, into Eq. (46), and the induced current into Eq. (26), the field modulation is obtained. The results are given in Fig. 28. The left figure represents the case where 20 capacitors are working on the lower side of the chamber. The right figure gives the case where all the capacitors $(k=$ $100)$ are working. Comparing the results, which are given

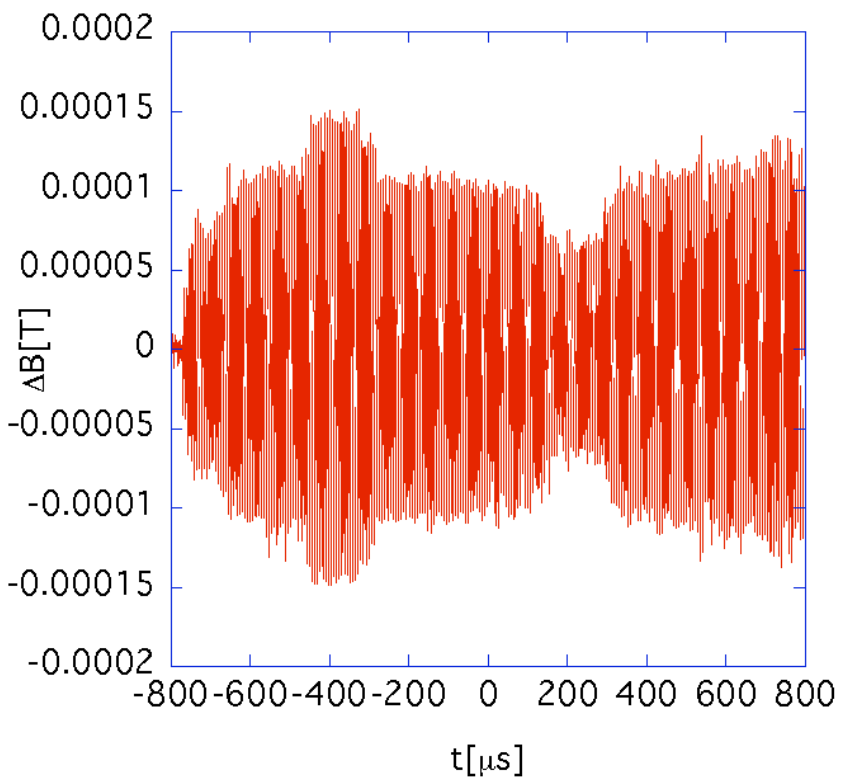

by red lines in Fig. 25, that correspond to the case where the original magnetic flux is excited of Eq. (28) with those given in Fig. 28 reveals that the behavior of those field modulations are quite different. Nevertheless, some of the phenomena can be understood through analysis of the previous simpler model. For example, Fig. 28 shows that the time when the maximum modulation occurs is not necessarily the same for different numbers of working capacitors $k$. This is because whether the oscillation of

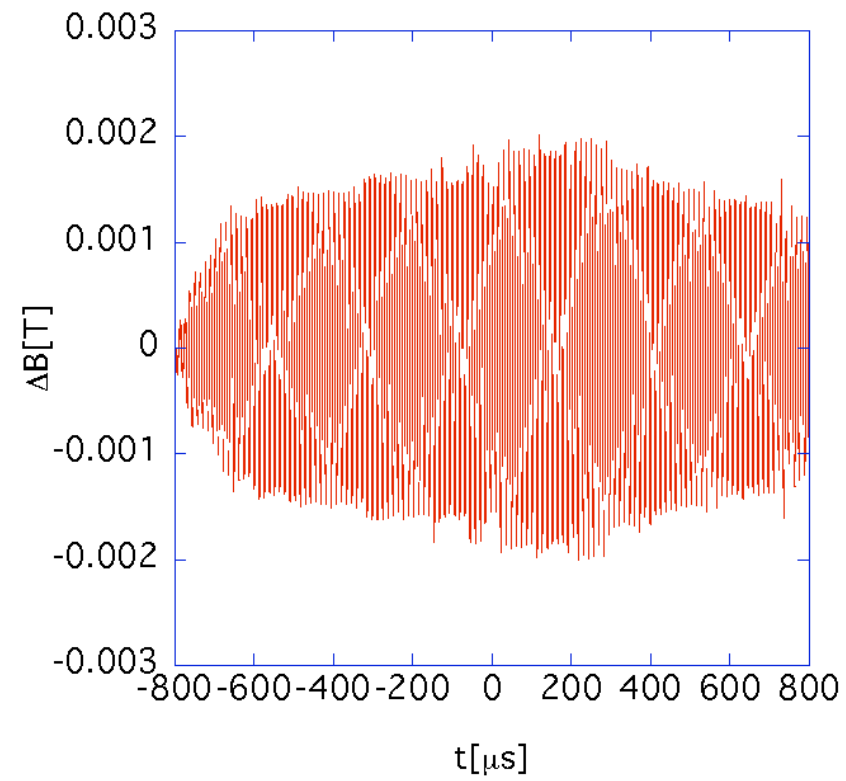

FIG. 28. (Color) The field modulation (theoretical results) due to the traverse of the area enclosed by different stripes by the measured magnetic flux (that is given by the left of Fig. 16). The left figure represents the case where 20 capacitors are working on the lower side of the chamber. The right figure represents the case where all the capacitors $(k=100)$ are working. 


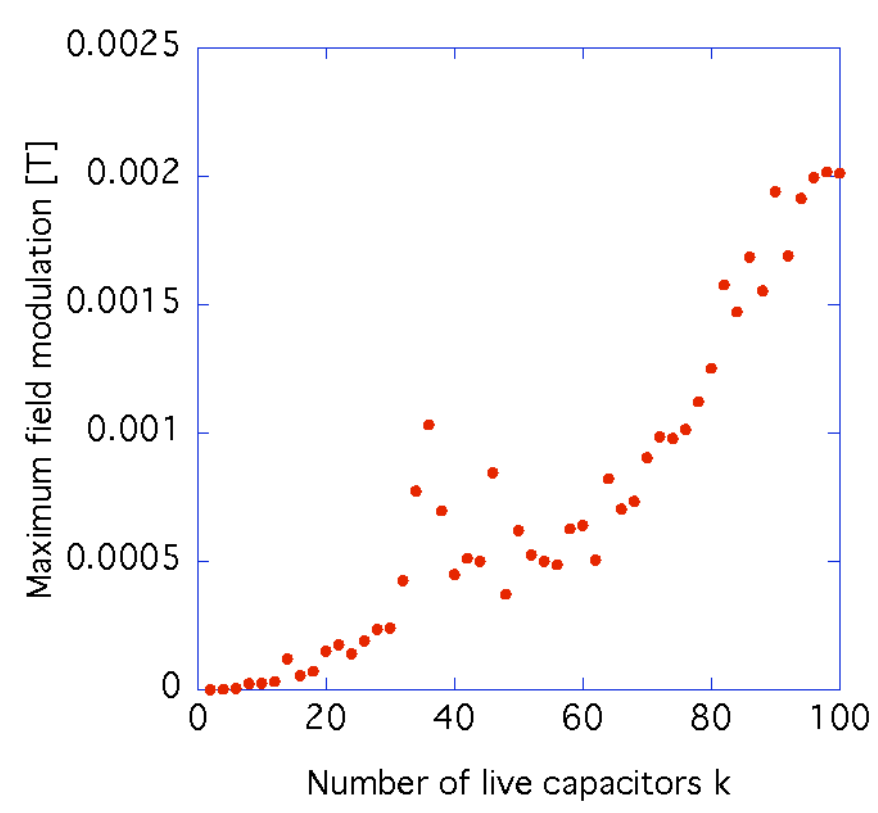

FIG. 29. (Color) The dependence of the maximum field modulation on the number of working capacitors $k$ when the measured field pattern (that is given by the left of Fig. 16) is excited.

the field modulation is enhanced or not depends on when the original magnetic flux kicks it, as discovered in the previous analysis. Furthermore, as given in Fig. 29 that describes the dependence of the maximum field modulation on the number of working capacitors $k$, the maximum field modulation does not necessarily become larger as $k$ does, although it is a rather broad tendency.

Figure 29 also reveals that the field modulation may be about $1.7 \%$ when compared to the original maximum value of $B(t)(\simeq 0.115 \mathrm{~T})$, if the chamber is completely surrounded by $\mathrm{Cu}$ stripes. In order to reduce the noise on the beam position monitors besides the chamber, or to the impedance source in terms of the beam instability issue, it is preferable that the chamber be completely surrounded by them, as mentioned in the Introduction. Since there is the broad tendency seen in Fig. 29 that the maximum field modulation becomes smaller as the number of stripes does, it is better to optimize to what degree the chamber should be surrounded by $\mathrm{Cu}$ stripes, from the view point of the design of accelerators.

The usefulness of Eq. (25) will now be discussed. In this discussion, the case where the magnetic flux $B(t)$ follows the more realistic field pattern given on the left of Fig. 16 is considered. The field modulation was calculated for the cases where the number of working capacitors $k$ was 20 , 60, and 100, respectively, using both Eqs. (25) and (26). The results are given in Fig. 30. The left, middle, and right figures give the cases where the number of working capacitors $k$ was 20,60 , and 100 , respectively. The blue points give the results calculated using Eq. (25) and the red points using Eq. (26). As there is a larger number of working capacitors, the approximation that the magnetic field on the $x=0$ plane is constant is appropriate, because the effect of the $\mathrm{Cu}$ stripes at both the right and left sides of the chamber dominates. Equations (25) and (26) are in good agreement of within a few percent for the case with the larger number of working capacitors. Hence, use of Eq. (25) saves on having to replace the yoke by image currents.

\section{B. The modulation of the magnetic field with a bending magnet in an RCS}

With an RCS, it is important to evaluate the COD in advance, because it cannot be corrected if the effect of it is too big, while with steering magnets it can be done if the amount of field error is tolerable. Since the strength of the magnet in an RCS changes over time, this phenomenon may cause a field modulation inside the chamber. The assumption was made that the magnetic flux changes as
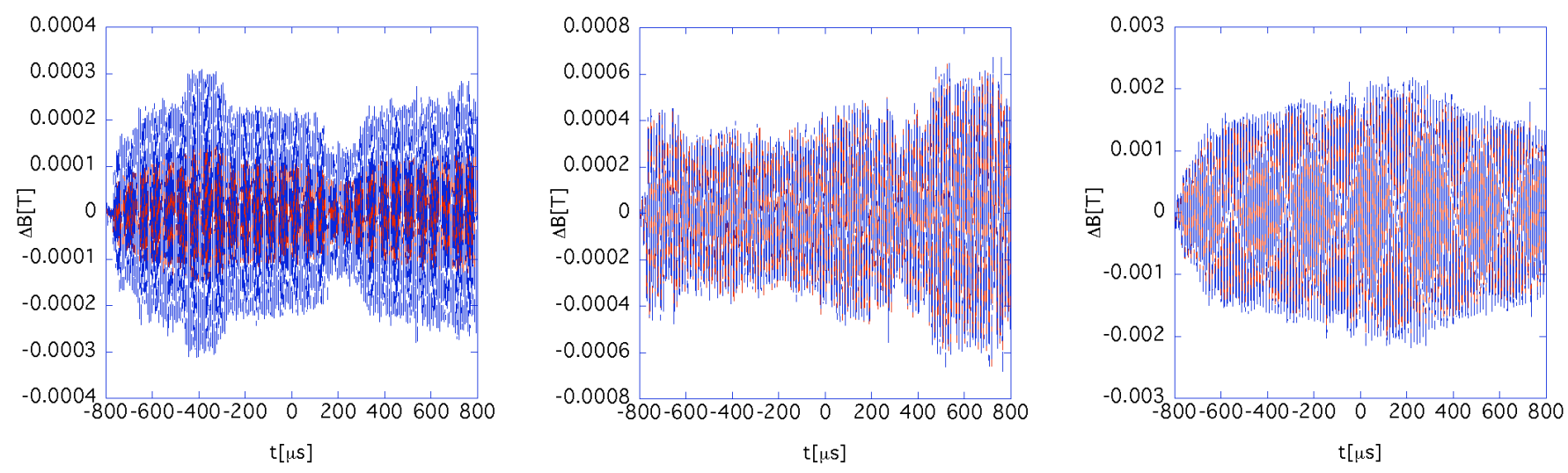

FIG. 30. (Color) The comparison of the field modulation (theoretical results) obtained by Eq. (25) with that done by Eq. (26) (when the original magnetic field is assumed to be excited, following the measured data that is given by the left of Fig. 16). The blue points represent the results calculated by Eq. (25) and red points do those done by Eq. (26). The left, middle, and right figures show the cases that the number of working capacitors $k$ is equal to 20,60, and 100, respectively. As the number of working capacitors is larger, the approximation that the magnetic field on $x=0$ plane is constant becomes appropriate. 

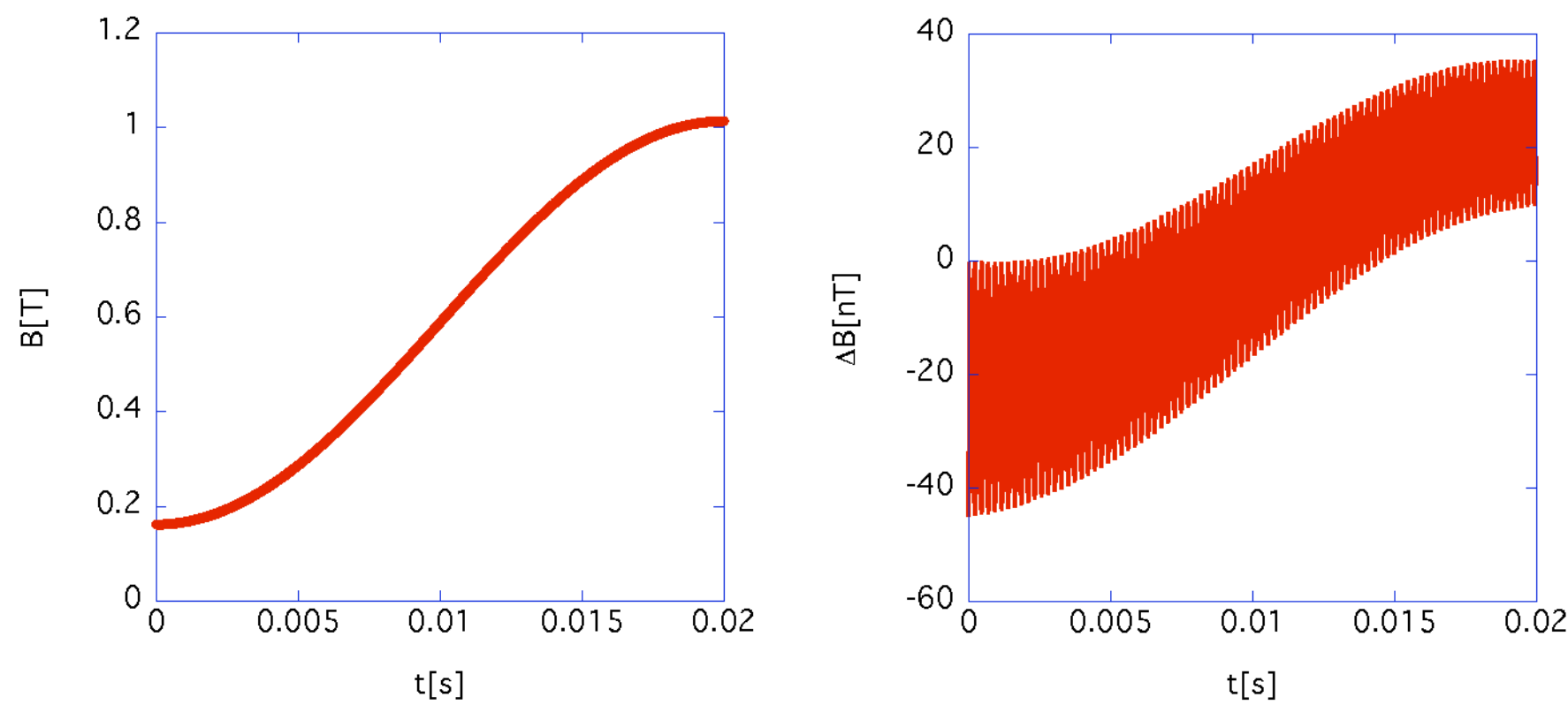

FIG. 31. (Color) The time dependence of the original bending magnet $B(t)$ is given in the left figure (analytical data) and the modulation of it (theoretical results) is in the right figure. The field modulation inside the chamber is insignificant. The same parameters as in Fig. 24 are used. Namely, the length of the chamber $l$ is $80 \mathrm{~cm}$, the radius of chamber $a$ is $15.25 \mathrm{~cm}$, the total number of $\mathrm{Cu}$ stripes $N$ is 100 , the attached capacitance $C$ for each stripe is $0.3 \mu \mathrm{F}$. The chamber is surrounded by the ferromagnetic yoke whose longitudinal length is $80 \mathrm{~cm}$, gap height $2 \mathrm{~g}=32.0 \mathrm{~cm}$, and gap width $2 \mathrm{~h}=32.0 \mathrm{~cm}$. As a typical set of parameters, ramping time $T=20 \mathrm{~ms}$, the radius of curvature $\rho=12.578 \mathrm{~m}$, the injection momentum $p_{\text {ini }}=0.61 \mathrm{GeV} / c$, and the extraction momentum $p_{\text {fin }}=3.8 \mathrm{GeV} / c$.

$$
B(t)=\frac{1}{\rho}\left(\frac{-p_{\mathrm{fin}} / c+p_{\mathrm{ini}} / c}{2} \cos \frac{\pi}{T} t+\frac{p_{\mathrm{ini}} / c+p_{\mathrm{fin}} / c}{2}\right),
$$

where $\rho$ is the radius of curvature of the bending magnet, $T$ the ramping time, and $p_{\text {ini }}$ and $p_{\text {fin }}$ are the injection and extraction momentum in units of $\mathrm{eV} / c$, respectively (refer to the left of Fig. 31). They are related to the total energy $E$ of the beam through the dispersion relation: $E^{2} / c^{2}-p^{2}=$ $m_{p}^{2} c^{2}$, where $m_{p}$ is the proton rest mass. Substituting the second differential of Eq. (47) with time $t$ into Eq. (24) results in the formula for the current $I_{i}$ on the $i$ th coil as

$$
\begin{aligned}
& I_{i}=-\sum_{j, k=1}^{N / 2} a l\left(p_{\text {fin }} / c-p_{\text {ini }} / c\right) \pi^{2} \frac{U_{i j} U_{j k}^{-1} \sin \theta_{k}}{2 \rho T^{2} \sqrt{R^{2}-\frac{2 \lambda_{j}}{C}}}\left[\frac{\left(\frac{R}{\lambda_{j}}+\frac{1}{\lambda_{j}} \sqrt{R^{2}-\frac{2 \lambda_{j}}{C}}\right)\left(\cos \frac{\pi}{T} t-e^{-\left[\left(R / \lambda_{j}\right)+\left(1 / \lambda_{j}\right) \sqrt{R^{2}-\left(2 \lambda_{j} / C\right)}\right] t}\right)+\frac{\pi}{T} \sin \frac{\pi}{T} t}{\left(\frac{R}{\lambda_{j}}+\frac{1}{\lambda_{j}} \sqrt{R^{2}-\frac{2 \lambda_{j}}{C}}\right)^{2}+\frac{\pi^{2}}{T^{2}}}\right. \\
& \left.-\frac{\left(\frac{R}{\lambda_{j}}-\frac{1}{\lambda_{j}} \sqrt{R^{2}-\frac{2 \lambda_{j}}{C}}\right)\left(\cos \frac{\pi}{T} t-e^{-\left[\left(R / \lambda_{j}\right)-\left(1 / \lambda_{j}\right) \sqrt{R^{2}-\left(2 \lambda_{j} / C\right)}\right]}\right)+\frac{\pi}{T} \sin \frac{\pi}{T} t}{\left(\frac{R}{\lambda_{j}}-\frac{1}{\lambda_{j}} \sqrt{R^{2}-\frac{2 \lambda_{j}}{C}}\right)^{2}+\frac{\pi^{2}}{T^{2}}}\right] .
\end{aligned}
$$

The modulation of the dipole field is calculated by substituting Eq. (48) into Eq. (26). As a typical example, the case can be considered where ramping time $T=20 \mathrm{~ms}$, the radius of curvature $\rho=12.578 \mathrm{~m}$, the injection momentum $p_{\text {ini }}=0.61 \mathrm{GeV} / c$, and the extraction momentum $p_{\text {fin }}=3.8 \mathrm{GeV} / c$. For the other parameters, the same values described in Fig. 24 are used. The results are given on the right in Fig. 31. Figure 32 gives the $N / 2$ normal mode frequencies: $1 / 2 \pi \sqrt{\lambda_{i} C}$ for this chamber. The modulation is seen to be insignificant. Since all the frequencies are within a few hundred $\mathrm{kHz}$, much higher than the inverse of ramping time $1 / T(=50 \mathrm{~Hz})$, the original magnetic field is considered to have been adiabatically excited when compared with the eigenmode frequencies of the coils.

\section{SUMMARY}

A theory was developed for use in evaluating the field modulation inside an rf-shielded chamber when the outside dipole magnetic field is time-dependently changed. Our 


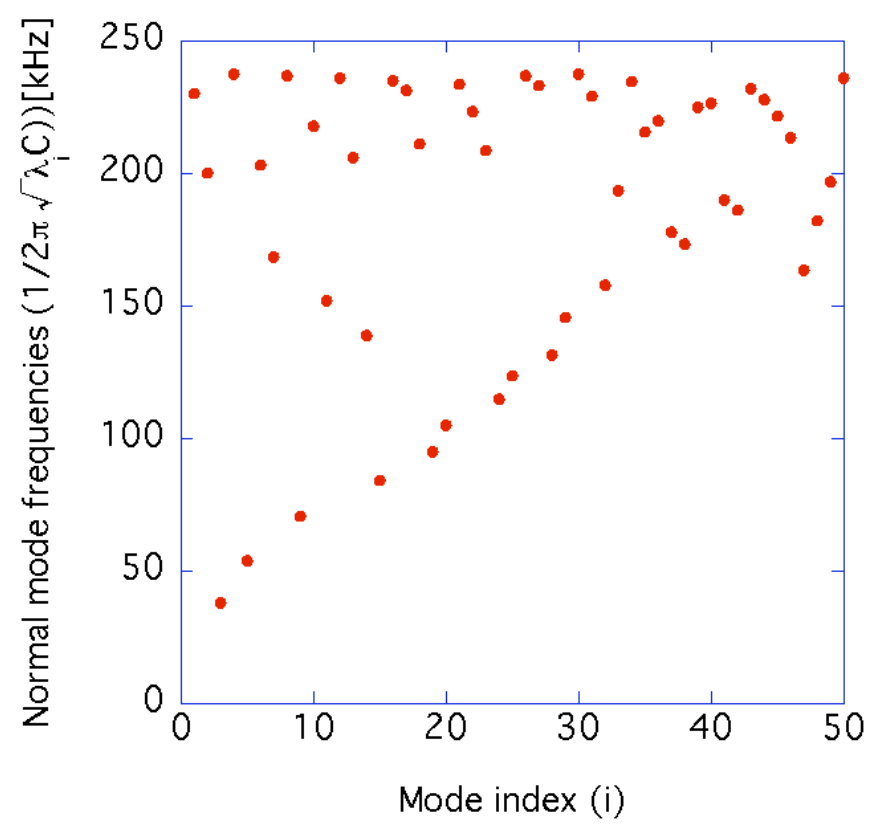

FIG. 32. (Color) The $N / 2$ normal mode frequencies $\left(1 / 2 \pi \sqrt{\lambda_{i} C}\right)$ where $i$ is mode index. These frequencies are much higher than the inverse of ramping time $1 / T(=50 \mathrm{~Hz})$. It may be considered that the original magnetic field is considered to have been adiabatically excited when compared with the eigenmode frequencies of the coils.

theory can predict the effect of the induced currents quickly and also estimate the field modulation inside the chamber within $6 \%-7 \%$ accuracy. This uncertainty is mainly caused by the fact that the original dipole magnetic field actually has a longitudinal component that leaks from the area where the magnet yoke is. The theory can be easily generalized to include the longitudinal effect if the 3D field map of the original magnetic field is known. This manipulation enables the field modulation inside the chamber to be calculated much more accurately.

The type of field modulation was classified into two by source: one from the traverse of the magnetic flux over a $\mathrm{Cu}$ stripe and the other from the traverse of magnetic flux over the area enclosed by a pair of $\mathrm{Cu}$ stripes and the flanges. If the ferromagnetic yoke was sufficiently far enough away from the chamber (typically 3 times more than the radius of the chamber), the field modulation due to the traverse of the magnetic flux over the $\mathrm{Cu}$ stripe would be almost canceled out, especially for the case of a symmetrical chamber (square or cylindrical chamber) covered in symmetrical stripes (square or cylindrical stripes). However in reality, the ferromagnetic yoke is very close to the chamber and the effect of it significant. The field modulation then, reaches a maximum when the chamber is completely surrounded by $\mathrm{Cu}$ stripes. Even in this case, the field modulation from the magnetic flux traversing the $\mathrm{Cu}$ stripe is smaller (typically less than the order of magnitude) than that caused in the traverse of the area enclosed by a pair of $\mathrm{Cu}$ stripes and the flanges.
The rf-shielded chamber was simplified as being a multicoil system in our theory to consider the effect of the magnetic flux traversing the area enclosed by a pair of $\mathrm{Cu}$ stripe and the flanges. This simplification proved to be applicable in this case by comparing theoretical results with simulated results. It is important to consider the effect of the mutual inductance between coils. The effect of it enhances the amount of field modulation because the damping time of the induced current is longer. The discovery was also made that the simpler formula given by Eq. (25) is very useful in evaluating the field modulation at the axis of the chamber, especially when the ferromagnetic yoke surrounds it. Infinite image currents are unnecessary, using that formula being considered.

As typical examples, the procedure was applied in two cases: the bump magnet during the injection period and the bending magnet in the RCS. We were able to determine a field pattern for the bump magnet so that the field modulation caused by it does not deteriorate the characteristics of the beam. From the point of view of the design of accelerators, the degree to which the chamber in the bump magnet should be surrounded by $\mathrm{Cu}$ stripes in reducing the field modulation could be optimized, while the noise on the beam position monitors, or the impedance source could be sufficiently small. Another discovery was that the field modulation is negligibly small inside the bending magnet in the RCS, because the bending magnet field is considered to have been adiabatically excited when compared with the intrinsic frequencies of an rf-shielded chamber.

\section{ACKNOWLEDGMENTS}

The authors would like to thank Y. H. Chin, K. Takata, T. Toyama, K. Oide, K. Yokoya, Y. Kamiya, T. Koseki, H. Hotchi, F. Noda, P. K. Saha, and H. Harada for their fruitful discussions. The authors also thank T. Takayanagi for kindly providing the data on the field patterns of the magnetic flux during the injection period with the $3 \mathrm{GeV}$ RCS at J-PARC. The authors also thank the members of the J-PARC project at JAEA/KEK.

\section{APPENDIX A: VALIDITY OF THE REPLACEMENT OF THE FERROMAGNETIC YOKE BY IMAGE CURRENTS}

In the text, we mentioned that the effect of the ferromagnetic yoke on the field modulation can be included in estimations by replacing it by image currents. In order to confirm that this was correct, we will show that the azimuthal component of this field modulation is zero on the magnetic pole face. This Appendix is used to concretely do so using the case where the field modulation is induced by the magnetic flux traversing the $\mathrm{Cu}$ stripe, as an example. A similar method can also be applied to the case where it is induced by the magnetic flux traversing $\mathrm{Cu}$ coils. 
The field modulation at $(x, y)$ due to the $i$ th stripe can be expressed using

$$
\begin{aligned}
H_{i}(x, y)= & \frac{2 c}{\pi b^{2} Z_{0}} \int_{-\infty}^{t} d t^{\prime} \frac{d B\left(t^{\prime}\right)}{d t^{\prime}} e^{\left[(4 c) /\left(Z_{0} b^{2} \sigma\right)\right]\left(t^{\prime}-t\right)} \sum_{m, n=-\infty}^{\infty}(-1)^{m} \\
& \times \int_{0}^{b} d r \oint d \varphi \frac{r^{2} \sin \varphi\left[-y+2 n g+(-1)^{n} y_{i}+r \cos \varphi, x-2 m h-(-1)^{m} x_{i}-r \sin \varphi, 0\right]}{\left[x-2 m h-(-1)^{m} x_{i}-r \sin \varphi\right]^{2}+\left[-y+2 n g+(-1)^{n} y_{i}+r \cos \varphi\right]^{2}},
\end{aligned}
$$

with image currents. It is sufficient to show only that the azimuthal component of $H_{i}$ is zero on the magnetic pole face, because the total magnetic field is expressed by the superposition of all the stripes. As shown in Fig. 3, since the gap height and gap width is $2 g$ and $2 h$, respectively, the field modulation on the magnetic pole face is denoted with that on the surfaces $:(x= \pm h, y)$ and $(x, y= \pm g)$, where $x$ and $y$ moves from $-h$ to $h$ and $-g$ to $g$, respectively.

As a typical case, we prove that the $x$ component of $H_{i}(x,-g)$ is zero. The component of $H_{i, x}(x,-g)$ can be expressed using

$$
\begin{aligned}
H_{i, x}(x,-g)= & \frac{2 c}{\pi b^{2} Z_{0}} \int_{-\infty}^{t} d t^{\prime} \frac{d B\left(t^{\prime}\right)}{d t^{\prime}} e^{\left[(4 c) /\left(Z_{0} b^{2} \sigma\right)\right]\left(t^{\prime}-t\right)} \sum_{m, n=-\infty}^{\infty}(-1)^{m} \\
& \times \int_{0}^{b} d r \oint d \varphi \frac{r^{2} \sin \varphi\left[g+2 n g+(-1)^{n} y_{i}+r \cos \varphi\right]}{\left[x-2 m h-(-1)^{m} x_{i}-r \sin \varphi\right]^{2}+\left[g+2 n g+(-1)^{n} y_{i}+r \cos \varphi\right]^{2}}
\end{aligned}
$$

By replacing index $n$ by $n-1$ and variable $\varphi$ by $\varphi-\pi$, it can be rewritten as

$$
\begin{aligned}
H_{i, x}(x,-g)= & \frac{2 c}{\pi b^{2} Z_{0}} \int_{-\infty}^{t} d t^{\prime} \frac{d B\left(t^{\prime}\right)}{d t^{\prime}} e^{\left[(4 c) /\left(Z_{0} b^{2} \sigma\right)\right]\left(t^{\prime}-t\right)} \sum_{m, n=-\infty}^{\infty}(-1)^{m} \\
& \times \int_{0}^{b} d r \oint d \varphi \frac{-r^{2} \sin \varphi\left[-g+2 n g-(-1)^{n} y_{i}-r \cos \varphi\right]}{\left[x-2 m h-(-1)^{m} x_{i}+r \sin \varphi\right]^{2}+\left[-g+2 n g-(-1)^{n} y_{i}-r \cos \varphi\right]^{2}} .
\end{aligned}
$$

Finally, by transforming index $n$ to $-n$ and variable $\varphi$ to $-\varphi$, the following is obtained:

$$
\begin{aligned}
H_{i, x}(x,-g)= & \frac{2 c}{\pi b^{2} Z_{0}} \int_{-\infty}^{t} d t^{\prime} \frac{d B\left(t^{\prime}\right)}{d t^{\prime}} e^{\left[(4 c) /\left(Z_{0} b^{2} \sigma\right)\right]\left(t^{\prime}-t\right)} \sum_{m, n=-\infty}^{\infty}(-1)^{m} \\
& \times \int_{0}^{b} d r \oint d \varphi \frac{-r^{2} \sin \varphi\left[g+2 n g+(-1)^{n} y_{i}+r \cos \varphi\right]}{\left[x-2 m h-(-1)^{m} x_{i}-r \sin \varphi\right]^{2}+\left[g+2 n g+(-1)^{n} y_{i}+r \cos \varphi\right]^{2}} .
\end{aligned}
$$

Comparing the description of $H_{i, x}(x,-g)$ in Eq. (A2) with that of Eq. (A4), we find that $H_{i, x}(x,-g)$ must satisfy the relation

$$
H_{i, x}(x,-g)=-H_{i, x}(x,-g)
$$

which means that $H_{i, x}(x,-g)$ is equal to zero. Similar to the previous method, we were also able to prove the other conditions: $H_{i, x}(x, g)=0$ and $H_{i, y}( \pm h, y)=0$.

[1] http://j-parc.jp/index-e.html.

[2] http://neutrons.ornl.gov/aboutsns/aboutsns.shtml.

[3] http://www.isis.rl.ac.uk.

[4] I. Sakai, Y. Arakida, I. Sugai, Y. Takeda, S. Machida, Y. Irie, T. Shimada, F. Noda, K. Shigaki, K. Yamamoto, Y. Watanabe, and Y. Ishi, in Proceedings of the 2002 European Particle Accelerator Conference, Paris, France (EPS-IGA/CERN, Geneva, 2002), p. 1040, http:// www.jacow.org/.
[5] D. Raparia, in Proceedings of the 21st Particle Accelerator Conference, Knoxville, Tennessee, 2005 (IEEE, Piscataway, NJ, 2005), p. 553, http://www.jacow. org/.

[6] B. Jones, D. Adams, and C. Warsop, in Proceedings of the 22nd Particle Accelerator Conference, Albuquerque, New Mexico, 2007 (IEEE, Albuquerque, New Mexico, 2007), p. 1640, http://www.jacow.org/.

[7] Y. H. Chin, S. Lee, K. Takata, T. Toyama, Y. Shobuda, and H. Tsutsui, in Proceedings of HB2006, Tsukuba, Japan, 2006, p. 125, http://www.jacow.org/.

[8] T. Hodges, R. Langstaff, C. Oram, M. Featherby, and C. Planner, in Proceedings of the 14th Particle Accelerator Conference, (APS Beam Physics), San Francisco, California, 1991 (IEEE, San Francisco, California, 1991), p. 2272, http://www.jacow.org/.

[9] T.F. Wang, Y.S. Kurennoy, and R. L. Gluckstern, Phys. Rev. ST Accel. Beams 4, 104201 (2001).

[10] Y. Shobuda, Y.H. Chin, K. Ohmi and T. Toyama, in Proceedings of the 21st Particle Accelerator Conference, 
Knoxville, Tennessee, 2005 (IEEE, Piscataway, NJ, 2005), p. 1898, http://www.jacow.org/.

[11] Y. Shobuda, Y.H. Chin, and K. Takata, Phys. Rev. ST Accel. Beams 10, 044403 (2007).

[12] T. Takayanagi, T. Ueno, Y. Irie, M. Kinsho, O. Takeda, Y. Yamazaki, M. Yoshimoto, J. Kamiya, M. Watanabe, and M. Kuramochi, in Proceedings of the 10th European Particle Accelerator Conference, Edinburgh, Scotland,
2006 (EPS-AG, Edinburgh, Scotland, 2006), p. 1762, http://www.jacow.org/.

[13] OPERA-3D is developed by Vector Fields Limited, England.

[14] For example, S. A. Schelkunoff, Electromagnetic Waves (D. Van Nostrand Co., Inc., New York, 1943).

[15] For example, J.D. Jackson, Classical Electrodynamics (John Wiley \& Sons, New York, 1998).

[16] T. Takayanagi (private communication). 